\title{
Comprehensive registry of esophageal cancer in Japan, 2013
}

\author{
Masayuki Watanabe ${ }^{1}$ (C) Yuji Tachimori ${ }^{2} \cdot$ Tsuneo Oyama $^{3} \cdot$ Yasushi Toh $^{4} \cdot$ Hisahiro Matsubara $^{5} \cdot$ Masaki Ueno $^{6}$. \\ $K_{\text {Koji Kono }}{ }^{7}$. Takashi Uno ${ }^{8} \cdot$ Ryu Ishihara $^{9} \cdot$ Kei Muro $^{10} \cdot$ Hodaka Numasaki $^{11} \cdot$ Koji Tanaka $^{12}$. Soji Ozawa ${ }^{13}$. \\ Kentaro Murakami ${ }^{5}$. Shiyori Usune ${ }^{14} \cdot$ Arata Takahashi $^{14} \cdot$ Hiroaki Miyata $^{14}$. The Registration Committee for \\ Esophageal Cancer of the Japan Esophageal Society
}

Received: 1 September 2020 / Accepted: 18 September 2020 / Published online: 13 October 2020 (c) The Author(s) 2020

\begin{abstract}
Background Esophageal cancer is the eighth most common cause of cancer mortality in Japan. More than 11,000 people had died from esophageal cancer in 2018. The Japan Esophageal Society has collected the data on patients' characteristics, performed treatment, and outcomes annually.

Methods We analyzed the data of patients who had first visited the participating hospitals in 2013. In 2019, the data collection method was changed from an electronic submission to a web-based data collection using the National Clinical Database (NCD). Japanese Classification of Esophageal Cancer 10th by the Japan Esophageal Society (JES) and UICC TNM Classification 7 th were used for cancer staging

Results A total of 8019 cases were registered from 334 institutions in Japan. Squamous cell carcinoma and adenocarcinoma accounted for $87.8 \%$ and $6.3 \%$, respectively. The 5-year survival rates of patients treated using endoscopic resection, concurrent chemoradiotherapy, radiotherapy alone, or esophagectomy were $88.3 \%, 32.4 \%, 24.4 \%$, and $59.3 \%$, respectively. Esophagectomy was performed in 4910 cases. The operative and the hospital mortality rates were $0.77 \%$ and $1.98 \%$, respectively. The survival curves showed a good discriminatory ability both in the clinical and pathologic stages by the JES system. The 5-year survival rate of patients with pStage IV in the UICC classification that included patients with supraclavicular node metastasis was better than that of patients with pStage IVb in JES classification.

Conclusion We hope this report contributes to improving all aspects of the diagnosis and treatment of esophageal cancer in Japan.
\end{abstract}

Keywords Esophageal cancer $\cdot$ Esophagectomy $\cdot$ Endoscopic resection $\cdot$ Chemotherapy $\cdot$ Chemoradiotherapy

\section{Preface 2013}

We deeply appreciate the great contributions of many physicians in the registry of esophageal cancer cases. The Comprehensive Registry of Esophageal Cancer in Japan, 2013, was published here. In 2019, the data collection method was changed from an electronic submission to a web-based

These data were first made available on July 15, 2020, as the Comprehensive Registry of Esophageal Cancer in Japan, 2013.

The authors were members of the Registration Committee for Esophageal Cancer, the Japan Esophageal Society, and made great contribution to the preparation of this material.

Masayuki Watanabe

masayuki.watanabe@jfcr.or.jp

Extended author information available on the last page of the article data collection using the National Clinical Database (NCD). Personal information was replaced with individual management code inside each institute, and the NCD collected only anonymized information. The registry complies with the Act for the Protection of Personal Information.

We briefly summarized the Comprehensive Registry of Esophageal Cancer in Japan, 2013. Japanese Classification of Esophageal Cancer 10th by the Japan Esophageal Society (JES) [1] and UICC TNM Classification 7th [2] were used for cancer staging according to the subjected year. A total of 8019 cases were registered from 334 institutions in Japan. Tumor locations were cervical: 4.8\%, upper thoracic: $12.1 \%$, middle thoracic: $46.5 \%$, lower thoracic: $28.2 \%$ and EG junction: $7.9 \%$. Superficial carcinomas (Tis, T1a, T1b) were $38.6 \%$. As for the histologic type of biopsy specimens, squamous cell carcinoma and adenocarcinoma accounted 
for $87.8 \%$ and $6.3 \%$, respectively. Regarding clinical results, the 5-year survival rates of patients treated using endoscopic resection, concurrent chemoradiotherapy, radiotherapy alone, or esophagectomy were $88.3 \%, 32.4 \%, 24.4 \%$, and $59.3 \%$, respectively. The endoscopic submucosal dissection accounted for $91.6 \%$ of endoscopic resection. Esophagectomy was performed in 4910 cases. Concerning the approach used for esophagectomy, $43.0 \%$ of the cases were treated thoracoscopically. The operative mortality (within 30 days after surgery) was $0.77 \%$, and the hospital mortality was $1.98 \%$. The Kaplan-Meier survival curves diverged according to the N-grade both in the JES and the UICC classifications. The survival curves showed a good discriminatory ability both in the clinical and pathologic stages by the JES system. However, the survival of cStage IIB was better than those of IB and IIA, while the survival curves were almost identical between cStage IIIc and IV in the UICC system. Also, the survival curve of pStage IIA merged with that of IIB, and the survival of pStage IV was better than that of IIIC. The 5-year survival rate of patients with pStage IV in the UICC classification that included patients with supraclavicular node metastasis was better than that of patients with pStage IVb in JES classification.

We hope that this Comprehensive Registry of Esophageal Cancer in Japan for 2013 will help to improve all aspects of the diagnosis and treatment of esophageal cancer in Japan.

\section{Contents}

I. Clinical factors of esophageal cancer patients treated in 2013

1. Institution-registered cases in 2013

2. Patient background

- Table 1 Age and gender

- Table 2 Primary treatment

- Table 3 Tumor location

- Table 4 Histologic types of biopsy specimens

- Table 5 Depth of tumor invasion, cT (UICC TNM 7th)

- Table 6 Lymph node metastasis, cN (UICC TNM 7th)

- Table 7 Distant metastasis, cM (UICC TNM 7th)

- Table 8 Clinical stage (UICC TNM 7th)

II. Results of endoscopically treated patients in $\mathbf{2 0 1 3}$

Table 9 Details of endoscopic treatment for curative intent

Table 10 Complications of EMR/ESD
Table 11 Pathological depth of tumor invasion of EMR/ESD specimens

Figure 1 Survival of patients treated with EMR/ ESD

Figure 2 Survival of patients treated with EMR/ ESD according to the pathological depth of tumor invasion, pT (JES 10th)

Figure 3 Survival of patients treated with EMR/ ESD according to the lymphatic and venous invasion

III. Results in patients treated with chemotherapy and/or radiotherapy in 2013

Table 12 Dose of irradiation (non-surgically treated cases)

Table 13 Dose of irradiation (surgically treated cases)

Figure 4 Survival of patients treated with chemotherapy and/or radiotherapy

Figure 5 Survival of patients treated with definitive chemoradiotherapy according to clinical stage (UICC TNM 7th)

Figure 6 Survival of patients underwent radiotherapy alone according to clinical stage (UICC TNM 7th)

IV. Results in patients who underwent esophagectomy in 2013

Table 14 Treatment modalities of esophagectomy Table 15 Tumor location

Table 16 Approaches to tumor resection

Table 17 Video-assisted surgery

Table 18 Fields of lymph node dissection according to the location of the tumor

Table 19 Reconstruction route

Table 20 Organs used for reconstruction

Table 21 Histological classification

Table 22 Depth of tumor invasion, pT (JES 10th)

Table 23 Pathological grading of lymph node metastasis, pN (JES 10th)

Table 24 Pathological findings of lymph node metastasis, pN (UICC TNM 7th)

Table 25 Pathological findings of distant organ metastasis, pM (JES 10th)

Table 26 Residual tumor

Table 27 Causes of death

Figure 7 Survival of patients who underwent esophagectomy

Figure 8 Survival of patients who underwent esophagectomy according to clinical stage (JES 10th) 
Figure 9 Survival of patients who underwent esophagectomy according to clinical stage (UICC TNM 7th)

Figure 10 Survival of patients who underwent esophagectomy according to the depth of tumor invasion, pT (JES 10th)

Figure 11 Survival of patients who underwent esophagectomy according to lymph node metastasis (JES 10th)

Figure 12 Survival of patients who underwent esophagectomy according to lymph node metastasis (UICC TNM 7th)

Figure 13 Survival of patients who underwent esophagectomy according to pathological stage (JES 10th)

Figure 14 Survival of patients who underwent esophagectomy according to pathological stage (UICC TNM 7th)

Figure 15 Survival of patients who underwent esophagectomy according to residual tumor $(\mathbf{R})$

\section{Clinical features of esophageal cancer patients treated in 2013}

Institution-registered cases in 2013.

Institutions

Ageo Central General Hospital

Aichi Cancer Center

Aichi Medical University Hospital

Aizawa Hospital

Akita University Hospital

Aomori Prefectural Central Hospital

Arao Municipal Hospital

Asahikawa Medical University Hospital

Cancer Institute Hospital of JFCR

Chiba Cancer Center

Chiba University Hospital

Chibaken Saiseikai Narashino Hospital

Chiba-Nishi General Hospital

Chigasaki Municipal Hospital

Chugoku Rosai Hospital

Dokkyo Medical University Hospital

Ehime Prefectural Central Hospital

Eijyu General Hospital

Fuchinobe General Hospital

Fuchu Hospital

Fujinomiya City General Hospital

Fujioka General Hospital

Fujisaki Hospital

Fujita Health University Hospital continued

Institutions

Fukaya Red Cross Hospital

Fukui Prefectural Hospital

Fukui University Hospital

Fukui-ken Saiseikai Hospital

Fukuoka City Hospital

Fukuoka Shin Mizumaki Hospital

Fukuoka University Chikushi Hospital

Fukuoka University Hospital

Fukuoka Wajiro Hospital

Fukushima Medical University Hospital

Fukuyama City Hospital

Gifu Prefectural General Center

Gifu University Hospital

Gunma Prefectural Cancer Center

Gunma Saiseikai Maebashi Hospital

Gunma University Hospital

Hachinohe City Hospital

Hakodate City Hospital

Hakodate Goryokaku Hospital

Hakodate National Hospital

Hamamatsu University Hospital

Heartlife Hospital

Higashiosaka City Medical Center

Hiraka General Hospital

Hiratsuka City Hospital

Hiratsuka Kyosai Hospital

Hirosaki University Hospital

Hiroshima City Asa Hospital

Hiroshima City Hospital

Hiroshima Red Cross Hospital \& Atomic-bomb Survivors Hospital

Hiroshima University Hospital

Hitachi General Hospital

Hofu Institute of Gastroenterology

Hokkaido University Hospital

Hospital of the University of Occupational and Environmental Health, Japan

Hyogo Cancer Center

Hyogo Prefectural Amagasaki General Medical Center

Ibaraki Prefectural Central Hospital

Iizuka Hospital

International Goodwill Hospital

International University of Health and Welfare Atami Hospital

International University of Health and Welfare Hospital

International University of Health and Welfare Ichikawa Hospital

International University of Health and Welfare Mita Hospital

Isehara Kyodo Hospital

Ishikawa Prefectural Central Hospital

Itami City Hospital

Iwata City Hospital

Iwate Medical University Hospital 


\section{continued}

Institutions

Iwate Prefectural Central Hospital

Iwate Prefectural Chubu Hospital

JA Hiroshima General Hospital

JA Kouseiren Enshu Hospital

JA Onomichi General Hospital

Japanese Red Cross Ashikaga Hospital

Japanese Red Cross Fukuoka Hospital

Japanese Red Cross Ishinomaki Hospital

Japanese Red Cross Kitami Hospital

Japanese Red Cross Kyoto Daiichi Hospital

Japanese Red Cross Maebashi Hospital

Japanese Red Cross Medical Center

Japanese Red Cross Musashino Hospital

Japanese Red Cross Nagasaki Genbaku Hospital

Japanese Red Cross Nagoya Daiichi Hospital

Japanese Red Cross Saitama Hospital

Japanese Red Cross Society Nagano Hospital

Japanese Red Cross Tottori Hospital

Japanese Red Cross Wakayama Medical Center

JCHO Gunma Chuo Hospital

JCHO Kyushu Hospital

JCHO Miyazaki Konan Hospital

JCHO Osaka Hospital

JCHO Saitama Medical Center

JCHO Tokuyama Central Hospital

JCHO Yokohama Chuo Hospital

Jichi Medical University Hospital

Jichi Medical University Saitama Medical Center

Juntendo University Hospital

Juntendo University Shizuoka Hospital

Juntendo University Urayasu Hospital

Junwakai Memorial Hospital

Kagawa Prefectural Central Hospital

Kagawa Rosai Hospital

Kagawa University Hospital

Kagoshima University Hospital

Kaizuka City Hospital

Kakogawa Central City hospital

Kanagawa Cancer Center

Kanazawa Medical University Hospital

Kanazawa University Hospital

Kansai Denryoku Hospital

Kansai Medical University Hospital

Kansai Medical University Medical Center

Kansai Rosai Hospital

Kanto Central Hospital

Kashiwa Kousei General Hospital

Kasugai Municipal Hospital

Kawasaki Hospital

Kawasaki Medical School Hospital continued

Institutions

Kawasaki Medical School Kawasaki Hospital

Kawasaki Municipal Hospital

Kawasaki Municipal Ida Hospital

Kawasaki Saiwai Hospital

Keio University Hospital

Keiyukai Sapporo Hospital

Kindai University Hospital

Kindai University Nara Hospital

Kinki Central Hospital

Kiryu Kousei General Hospital

Kitaakita Municipal Hospital

Kitaharima Medical Center

Kitakyushu General Hospital

Kitakyushu Municipal Medical Center

Kitano Hospital

Kitasato University Hospital

Kobe City Medical Center General Hospital

Kobe University Hospital

Kochi Health Science Center

Kochi University Hospital

Kokura Memorial Hospital

Kouseiren Takaoka Hospital

Kumagai General Hospital

Kumamoto University Hospital

Kummoto Regional Medical Center

Kurashiki Central Hospital

Kurume University Hospital

Kyorin University Hospital

Kyoto University Hospital

Kyoto-Katsura Hospital

Kyushu Central Hospital

Kyushu University Hospital

Machida Municipal hospital

Matsudo City General Hospital

Matsushita Memorial Hospital

Matsuyama Red Cross Hospital

Mie University Hospital

Minamiosaka Hospital

Minoh City Hospital

Mito Red Cross Hospital

Mitsui Memorial Hospital

Miyazaki University Hospital

Mizushima Kyudo Hospital

Moriguchi Keijinkai Hospital

Murakami General Hospital

Nagahama City Hospital

Nagahama Red Cross Hospital

Nagano Municipal Hospital

Nagaoka Chuo General Hospital

Nagasaki University Hospital 
continued

\section{Institutions}

Nagoya City University Hospital

Nagoya City West Medical Center

Nagoya University Hospital

Nanpuh Hospital

Nara City Hospital

Nara Medical University Hospital

Nasu Red Cross Hospital

National Cancer Center Hospital

National Cancer Center Hospital East

National Center for Global Health and Medicine

National Defence Medical College Hospital

New Tokyo Hospital

NHO Beppu Medical Center

NHO Chiba Medical Center

NHO Fukuoka-Higashi Medical Center

NHO Iwakuni Clinincal Center

NHO Kanmon Medical Center

NHO Kure Medical Center

NHO Kyoto Medical Center

NHO Kyushu Cancer Center

NHO Matsumoto Medical Center

NHO Mito Medical Center

NHO Miyakonojo Medical Center

NHO Nagasaki Medical Center

NHO Nagoya Medical Center

NHO Okayama Medical Center

NHO Osaka Medical Center

NHO Saitama Hospital

NHO Sendai Medical Center

NHO Shikoku Cancer Center

NHO Tokyo Medical Center

NHO Yokohama Medical Center

Nihonkai General Hospital

Niigata Cancer Center Hospital

Niigata City General Hospital

Niigata Prefectural Central Hospital

Niigata Prefectural Shibata Hospital

Niigata University Medical \& Detal Hospital

Nikko Memorial Hospital

Nippon Medical School Chiba Hokusou Hospital

Nippon Medical School Hospital

Nippon Medical School Musashi Kosugi Hospital

Nippon Medical School Tama Nagayama Hospital

Nishi Kobe Medical Center

Nissan Tamagawa Hospital

Nozaki Tokushukai Hospital

Numazu City Hospital

Obihiro Kousei Hospital

Ogaki Municipal Hospital

Ohta Hospital continued

Institutions

Ohta Nishinouchi Hospital

Oita Red Cross Hospital

Oita University Hospital

Okayama Red Cross General Hospital

Okayama Saiseikai General Hospital

Okayama University Hospital

Okitama Public General Hospital

Onomichi Municipal Hospital

Osaka City General Hospital

Osaka City University Hospital

Osaka Ekisaikai Hospital

Osaka General Medical Center

Osaka International Cancer Institute

Osaka Medical College Hospital

Osaka Police Hospital

Osaka Red Cross Hospital

Osaki City Hospital

Otsu City Hospital

Rinku General Medical Center

Saga Prefectural Hospital Koseikan

Saga University Hospital

Sagamihara National Hospital

Saiseikai Fukuoka General Hospital

Saiseikai Karatsu Hospital

Saiseikai Noe Hospital

Saiseikai Utsunomiya Hospital

Saiseikai Yokohama Tobu Hospital

Saitama Cancer Center

Saitama Medical University International Medical Center

Saitama Medical University Saitama Medical Center

Sakai City Medical Center

Saku Central Hospital

Sapporo Medical University Hospital

Seikei-kai Chiba Medical Center

Sendai City Hospital

Shiga General Hospital

Shiga University of Medical Science Hospital

Shimane University Hospital

Shin Takeo Hospital

Shinko Hospital

Shinshu University Hospital

Shizuoka Cancer Center

Shizuoka City Shizuoka Hospital

Shizuoka General Hospital

Shizuoka Saiseikai General Hospital

Showa University Hospital

Southern Tohoku General Hospital

St. Luke's International Hospital

St. Marianna University School of Medicine Hospital

St. Mary's Hospital 


\section{continued}

Institutions

Steel Memorial Yawata Hospital

Suita Municipal Hospital

Suzuka Chuo General Hospital

Tachikawa Hospital

Takatsuki Red Cross Hospital

Teikyo University Chiba Medical Center

Teikyo University Hospital

Teine Keijinkai Hospital

Tenri Hospital

The Hospital of Hyogo College of Medicine

The Jikei University Daisan Hospital

The Jikei University Hospital

Tochigi Cancer Center

Toho University Ohashi Medical Center

Toho University Omori Medical Center

Toho University Sakura Medical Center

Tohoku University Hospital

Tokai University Hachioji Hospital

Tokai University Hospital

Tokai University Tokyo Hospital

Tokushima Red Cross Hospital

Tokushima University Hospital

Tokyo Dental College Ichikawa General Hospital

Tokyo Medical and Dental University Hospital

Tokyo Medical University Hachioji Medical Center

Tokyo Medical University Hospital

Tokyo Metropolitan Cancer and Infectious Diseases Center Komagome Hospital

Tokyo Metropolitan Tama Medical Center

Tokyo Rosai Hospital

Tokyo University Hospital

Tokyo Women's Medical University Hospital

Tokyo Women's Medical University Medical Center East

Tokyo Women's Medical University Yachiyo Medical Center

Tonan Hospital

Toranomon Hospital

Tosei General Hospital

Toshima Hospital

Tottori Prefectural Central Hospital

Tottori University Hospital

Toyama Prefectural Central Hospital

Toyama University Hospital

Toyonaka Municipal Hospital

Toyota Memorial Hospital

Tsuchiura Kyodo Hospital

Tsukuba University Hospital

Tsuruoka Municipal Shonal Hospital

Tsuyama Chuo Hospital continued

Institutions

University Hospital Kyoto Prefectural University of Medicine

University of the Ryukyus Hospital

Wakayama Medical University Hospital

Yamagata Prefectural Central Hospital

Yamagata University Hospital

Yamaguchi University Hospital

Yamanashi Prefectural Central Hospital

Yamanashi University Hospital

Yao Municipal Hospital

Yokohama City Municipal Hospital

Yokohama City University Hospital

Yokohama City University Medical Center

Yokohama Sakae Kyosai Hospital

Yokosuka General Hospital Uwamachi

(Total 334 institutions)

\section{Patient background}

Tables 1, 2, 3, 4, 5, 6, 7, 8

Table 1 Age and gender

\begin{tabular}{llll}
\hline Age & Male & Female & Cases $(\%)$ \\
\hline$\leq 29$ & 12 & 1 & $13(0.2 \%)$ \\
$30-39$ & 16 & 6 & $22(0.3 \%)$ \\
$40-49$ & 164 & 59 & $223(2.8 \%)$ \\
$50-59$ & 917 & 174 & $1091(13.6 \%)$ \\
$60-69$ & 2675 & 431 & $3106(38.7 \%)$ \\
$70-79$ & 2403 & 437 & $2840(35.4 \%)$ \\
$80-89$ & 570 & 133 & $703(8.8 \%)$ \\
$90 \leq$ & 10 & 11 & $21(0.3 \%)$ \\
Total & 6767 & 1252 & 8019 \\
\hline
\end{tabular}

Table 2 Performed treatment

\begin{tabular}{ll}
\hline Treatments & Cases (\%) \\
\hline Surgery & $5038(62.8 \%)$ \\
Esophagectomy & $4910(61.2 \%)$ \\
Palliative surgery & $128(1.6 \%)$ \\
Chemotherapy and/or Radiotherapy & $4062(50.7 \%)$ \\
Endoscopic treatment & $1421(17.7 \%)$ \\
\hline
\end{tabular}


Table 3 Tumor location

\begin{tabular}{|c|c|c|c|c|c|}
\hline \multirow[t]{2}{*}{ Location of tumor } & \multirow{2}{*}{$\begin{array}{l}\text { Endoscopic treatment } \\
(\%)\end{array}$} & \multicolumn{2}{|l|}{ Surgery } & \multirow{2}{*}{$\begin{array}{l}\text { Chemotherapy and/or } \\
\text { radiotherapy (\%) }\end{array}$} & \multirow[t]{2}{*}{ Total $(\%)$} \\
\hline & & Esophagectomy (\%) & Palliative surgery (\%) & & \\
\hline Cervical & $48(3.4 \%)$ & $163(3.3 \%)$ & $10(7.8 \%)$ & $256(6.3 \%)$ & $384(4.8 \%)$ \\
\hline Upper thoracic & $142(10.0 \%)$ & $525(10.7 \%)$ & $25(19.5 \%)$ & $597(14.7 \%)$ & $969(12.1 \%)$ \\
\hline Middle thoracic & $775(54.5 \%)$ & $2188(44.6 \%)$ & $61(47.7 \%)$ & $1864(45.9 \%)$ & $3726(46.5 \%)$ \\
\hline Lower thoracic & $369(26.0 \%)$ & $1544(31.4 \%)$ & $26(20.3 \%)$ & $1118(27.5 \%)$ & $2264(28.2 \%)$ \\
\hline EG & $61(4.3 \%)$ & $356(7.3 \%)$ & $5(3.9 \%)$ & $165(4.1 \%)$ & $470(5.9 \%)$ \\
\hline $\mathrm{E}=\mathrm{G}$ & $14(1.0 \%)$ & $66(1.3 \%)$ & & $23(0.6 \%)$ & $88(1.1 \%)$ \\
\hline GE & $6(0.4 \%)$ & $61(1.2 \%)$ & & $15(0.4 \%)$ & $72(0.9 \%)$ \\
\hline Unknown & $6(0.4 \%)$ & $7(0.1 \%)$ & $1(0.8 \%)$ & $45(0.6 \%)$ & $46(0.6 \%)$ \\
\hline Total & 1421 & 4910 & 128 & 4062 & 8019 \\
\hline
\end{tabular}

$E$ esophageal, $G$ gastric

Table 4 Histologic type of biopsy specimens

\begin{tabular}{|c|c|c|c|c|c|}
\hline \multirow[t]{2}{*}{ Histologic types } & \multirow{2}{*}{$\begin{array}{l}\text { Endoscopic treatment } \\
(\%)\end{array}$} & \multicolumn{2}{|l|}{ Surgery } & \multirow{2}{*}{$\begin{array}{l}\text { Chemotherapy and/or } \\
\text { radiotherapy (\%) }\end{array}$} & \multirow[t]{2}{*}{ Total $(\%)$} \\
\hline & & Esophagectomy (\%) & Palliative surgery $(\%)$ & & \\
\hline Squamous cell carcinoma & $1101(77.5 \%)$ & $4291(87.4 \%)$ & $116(90.6 \%)$ & $3733(91.9 \%)$ & $6911(86.2 \%)$ \\
\hline Squamous cell carcinoma & $867(61.0 \%)$ & $2442(49.7 \%)$ & $74(57.8 \%)$ & $2330(57.4 \%)$ & $4377(54.6 \%)$ \\
\hline Well differentiated & $100(7.0 \%)$ & $387(7.9 \%)$ & $9(7.0 \%)$ & $259(6.4 \%)$ & $565(7.0 \%)$ \\
\hline Moderately differentiated & $117(8.2 \%)$ & $1093(22.3 \%)$ & $24(18.8 \%)$ & $805(19.8 \%)$ & $1448(18.1 \%)$ \\
\hline Poorly differentiated & $17(1.2 \%)$ & $369(7.5 \%)$ & $9(7.0 \%)$ & $339(8.3 \%)$ & $521(6.5 \%)$ \\
\hline Adenocarcinoma & $38(2.7 \%)$ & $340(6.9 \%)$ & $4(3.1 \%)$ & $133(3.3 \%)$ & $419(5.2 \%)$ \\
\hline Barrett's carcinoma & $33(2.3 \%)$ & $91(1.9 \%)$ & $1(0.8 \%)$ & $25(0.6 \%)$ & $133(1.7 \%)$ \\
\hline Adenosquamous carcinoma & $2(0.1 \%)$ & $11(0.2 \%)$ & & $4(0.1 \%)$ & $14(0.2 \%)$ \\
\hline Mucoepidermoid carcinoma & & $1(0.0 \%)$ & & & $1(0.0 \%)$ \\
\hline Basaloid carcinoma & $2(0.1 \%)$ & $31(0.6 \%)$ & & $16(0.4 \%)$ & $39(0.5 \%)$ \\
\hline Neuroendocrine tumor & & $1(0.0 \%)$ & & $1(0.0 \%)$ & $2(0.0 \%)$ \\
\hline Neuroendocrine carcinoma & $4(0.3 \%)$ & $21(0.4 \%)$ & & $27(0.7 \%)$ & $34(0.4 \%)$ \\
\hline Undifferentiated carcinoma & & $6(0.1 \%)$ & & $4(0.1 \%)$ & $9(0.1 \%)$ \\
\hline Malignant melanoma & & $15(0.3 \%)$ & & $6(0.1 \%)$ & $19(0.2 \%)$ \\
\hline Carcinosarcoma & & $16(0.3 \%)$ & $1(0.8 \%)$ & $8(0.2 \%)$ & $19(0.2 \%)$ \\
\hline GIST & & $1(0.0 \%)$ & & & $1(0.0 \%)$ \\
\hline Adenoid cystic carcinoma & & $1(0.0 \%)$ & & & $1(0.0 \%)$ \\
\hline Nonepithelial tumors & $2(0.1 \%)$ & & & & $3(0.0 \%)$ \\
\hline Other epithelial tumors & $17(1.2 \%)$ & $4(0.1 \%)$ & & $8(0.2 \%)$ & $27(0.3 \%)$ \\
\hline Other tumors & $51(3.6 \%)$ & $16(0.3 \%)$ & & $13(0.3 \%)$ & $79(1.0 \%)$ \\
\hline Unknown & $171(12.0 \%)$ & $64(1.3 \%)$ & $6(4.7 \%)$ & $84(2.1 \%)$ & $308(3.8 \%)$ \\
\hline Total & 1421 & 4910 & 128 & 4062 & 8019 \\
\hline
\end{tabular}


Table 5 Depth of tumor invasion, cT (UICC TNM 7th)

\begin{tabular}{|c|c|c|c|c|c|}
\hline \multirow[t]{2}{*}{ Clinical T } & \multirow{2}{*}{$\begin{array}{l}\text { Endoscopic treatment } \\
\text { (\%) }\end{array}$} & \multicolumn{2}{|l|}{ Surgery } & \multirow{2}{*}{$\begin{array}{l}\text { Chemotherapy and/or } \\
\text { radiotherapy (\%) }\end{array}$} & \multirow[t]{2}{*}{ Total $(\%)$} \\
\hline & & Esophagectomy (\%) & Palliative surgery (\%) & & \\
\hline cTX & $12(0.8 \%)$ & $11(0.2 \%)$ & $1(0.8 \%)$ & $34(0.8 \%)$ & $80(1.0 \%)$ \\
\hline cT0 & $6(0.6 \%)$ & $4(0.1 \%)$ & & $3(0.1 \%)$ & $14(0.2 \%)$ \\
\hline cT1a & $1139(80.2 \%)$ & $247(5.0 \%)$ & & $102(2.5 \%)$ & $1426(17.8 \%)$ \\
\hline $\mathrm{cT} 1 \mathrm{~b}$ & $196(13.8 \%)$ & $1319(26.9 \%)$ & $9(7.0 \%)$ & $515(12.7 \%)$ & $1658(20.7 \%)$ \\
\hline cT2 & $4(0.3 \%)$ & $832(16.9 \%)$ & $4(3.1 \%)$ & $609(15.0 \%)$ & $1006(12.5 \%)$ \\
\hline cT3 & $41(2.9 \%)$ & $2223(45.3 \%)$ & $49(38.3 \%)$ & $2036(50.1 \%)$ & $2895(36.1 \%)$ \\
\hline cT4a & $4(0.3 \%)$ & $133(2.7 \%)$ & $17(13.3 \%)$ & $257(6.3 \%)$ & $341(4.3 \%)$ \\
\hline $\mathrm{cT} 4 \mathrm{~b}$ & $19(1.3 \%)$ & $141(2.9 \%)$ & $48(37.5 \%)$ & $506(12.5 \%)$ & $599(7.5 \%)$ \\
\hline Total & 1421 & 4910 & 128 & 4062 & 8019 \\
\hline
\end{tabular}

Table 6 Lymph node metastasis, cN (UICC TNM 7th)

\begin{tabular}{|c|c|c|c|c|c|}
\hline \multirow[t]{2}{*}{ Clinical N } & \multirow{2}{*}{$\begin{array}{l}\text { Endoscopic treatment } \\
(\%)\end{array}$} & \multicolumn{2}{|l|}{ Surgery } & \multirow{2}{*}{$\begin{array}{l}\text { Chemotherapy and/or } \\
\text { radiotherapy }(\%)\end{array}$} & \multirow[t]{2}{*}{ Total $(\%)$} \\
\hline & & Esophagectomy (\%) & Palliative surgery $(\%)$ & & \\
\hline $\mathrm{cN} 0$ & $1351(95.1 \%)$ & $2278(46.4 \%)$ & $31(24.2 \%)$ & $1117(27.5 \%)$ & $4047(50.5 \%)$ \\
\hline $\mathrm{cN} 1$ & $38(2.7 \%)$ & $1704(34.7 \%)$ & $37(28.9 \%)$ & $1663(40.9 \%)$ & $2318(28.9 \%)$ \\
\hline $\mathrm{cN} 2$ & $22(1.5 \%)$ & $800(16.3 \%)$ & $43(33.6 \%)$ & $1009(24.8 \%)$ & $1301(16.2 \%)$ \\
\hline $\mathrm{cN} 3$ & $10(0.7 \%)$ & $128(2.6 \%)$ & $17(13.3 \%)$ & $273(6.7 \%)$ & $353(4.4 \%)$ \\
\hline Total & 1421 & 4910 & 128 & 4062 & 8019 \\
\hline
\end{tabular}

Table 7 Distant metastasis, cM (UICC TNM 7th)

\begin{tabular}{|c|c|c|c|c|c|}
\hline \multirow[t]{2}{*}{ Clinical M } & \multirow{2}{*}{$\begin{array}{l}\text { Endoscopic treatment } \\
(\%)\end{array}$} & \multicolumn{2}{|l|}{ Surgery } & \multirow{2}{*}{$\begin{array}{l}\text { Chemotherapy and/or } \\
\text { radiotherapy }(\%)\end{array}$} & \multirow[t]{2}{*}{ Total (\%) } \\
\hline & & Esophagectomy (\%) & Palliative surgery $(\%)$ & & \\
\hline cM0 & $1406(98.9 \%)$ & $4753(96.8 \%)$ & $103(80.5 \%)$ & $3513(86.5 \%)$ & $7350(91.7 \%)$ \\
\hline cM1 & $15(1.1 \%)$ & $157(3.2 \%)$ & $25(19.5 \%)$ & $549(13.5 \%)$ & $669(8.3 \%)$ \\
\hline Total & 1421 & 4910 & 128 & 4062 & 8019 \\
\hline
\end{tabular}

Table 8 Clinical Stage (UICC TNM 7th)

\begin{tabular}{|c|c|c|c|c|c|}
\hline \multirow[t]{2}{*}{ Clinical stage } & \multirow{2}{*}{$\begin{array}{l}\text { Endoscopic treatment } \\
(\%)\end{array}$} & \multicolumn{2}{|l|}{ Surgery } & \multirow{2}{*}{$\begin{array}{l}\text { Chemotherapy and/or } \\
\text { radiotherapy (\%) }\end{array}$} & \multirow[t]{2}{*}{ Total $(\%)$} \\
\hline & & Esophagectomy (\%) & Palliative surgery (\%) & & \\
\hline Stage IA & $1317(92.7 \%)$ & $1268(25.8 \%)$ & $7(5.5 \%)$ & $388(9.6 \%)$ & $2712(33.8 \%)$ \\
\hline Stage IB & $3(0.2 \%)$ & $417(8.5 \%)$ & $3(2.3 \%)$ & $252(6.2 \%)$ & $492(6.1 \%)$ \\
\hline Stage IIA & $8(0.6 \%)$ & $523(10.7 \%)$ & $10(7.8 \%)$ & $356(8.8 \%)$ & $629(7.8 \%)$ \\
\hline Stage IIB & $15(1.1 \%)$ & $522(10.6 \%)$ & & $389(9.6 \%)$ & $609(7.6 \%)$ \\
\hline Stage IIIA & $19(1.3 \%)$ & $1169(23.8 \%)$ & $14(10.9 \%)$ & $952(23.4 \%)$ & $1375(17.1 \%)$ \\
\hline Stage IIIB & $9(0.6 \%)$ & $514(10.5 \%)$ & $14(10.9 \%)$ & $488(12.0 \%)$ & $642(8.0 \%)$ \\
\hline Stage IIIC & $17(1.2 \%)$ & $325(6.6 \%)$ & $55(43.0 \%)$ & $667(16.4 \%)$ & $821(10.2 \%)$ \\
\hline Stage IV & $15(1.1 \%)$ & $157(3.2 \%)$ & $25(19.5 \%)$ & $549(13.5 \%)$ & $669(8.3 \%)$ \\
\hline Unknown & $18(1.3 \%)$ & $15(0.3 \%)$ & & $21(0.5 \%)$ & $70(0.9 \%)$ \\
\hline Total & 1421 & 4910 & 128 & 4062 & 8019 \\
\hline
\end{tabular}




\section{Results of endoscopically treated patients in 2013}

Tables 9, 10, 11, and Figs. 1, 2, 3.

Table 9 Details of endoscopic treatment for curative intent

\begin{tabular}{ll}
\hline Treatment details & Cases (\%) \\
\hline EMR & $108(8.0 \%)$ \\
EMR + YAG laser & $1(0.1 \%)$ \\
EMR + MCT/RFA & \\
ESD & $1224(90.2 \%)$ \\
ESD + EMR & $4(0.3 \%)$ \\
ESD + PDT & \\
ESD + YAG laser & $5(0.4 \%)$ \\
PDT & $2(0.1 \%)$ \\
YAG laser & $13(1.0 \%)$ \\
Total & 1357 \\
\hline
\end{tabular}

$E M R$ endoscopic mucosal resection, $P D T$ photodynamic therapy, $Y A G$ yttrium aluminum garnet, $M C T$ microwave coagulation therapy, $E S D$ endoscopic submucosal dissection
Table 10 Complications of EMR/ESD

\begin{tabular}{ll}
\hline $\begin{array}{l}\text { Complications } \\
\text { of EMR/ESD }\end{array}$ & Cases (\%) \\
\hline None & $1298(92.7 \%)$ \\
Perforation & $10(0.7 \%)$ \\
Bleeding & $1(0.1 \%)$ \\
Mediastinitis & $1(0.1 \%)$ \\
Stenosis & $23(1.7 \%)$ \\
Others & \\
Unknown & $2(0.1 \%)$ \\
Total & 1335
\end{tabular}

Table 11 Pathologic depth of tumor invasion of MER/ESD specimens

\begin{tabular}{ll}
\hline Pathological depth of tumor invasion $(\mathrm{pT})$ & Cases $(\%)$ \\
\hline pTX & $22(1.6 \%)$ \\
pT0 & $7(0.5 \%)$ \\
pT1a & $1111(82.8 \%)$ \\
pT1b & $201(15.0 \%)$ \\
pT2 & \\
pT3 & $1(0.1 \%)$ \\
Total & 1342 \\
\hline
\end{tabular}

Fig. 1 Survival of patients treated with EMR/ESD

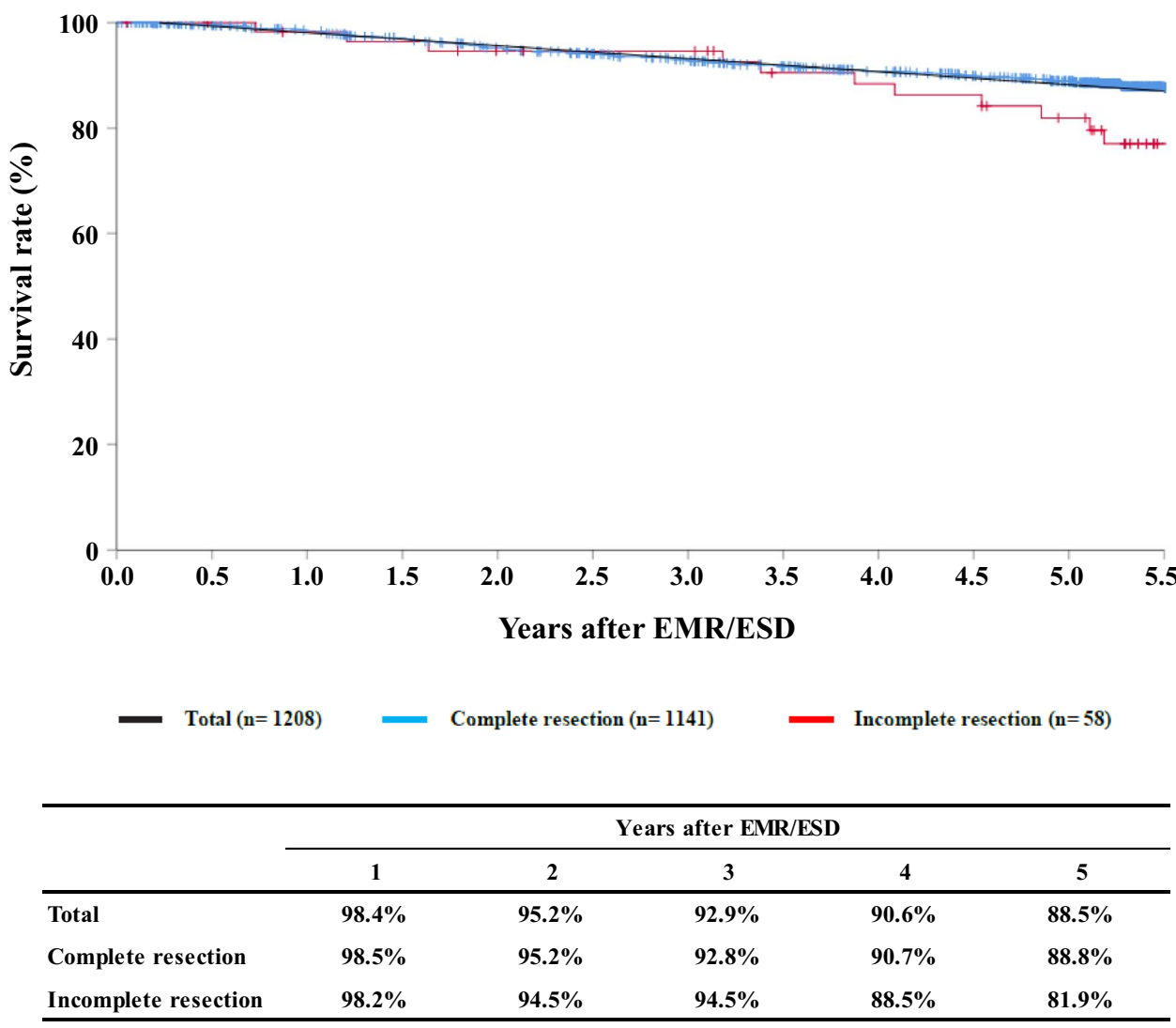


Fig. 2 Survival of patients treated with EM/ESD according to the pathological depth of tumor invasion, pT(JES 10th)

Fig. 3 Survival of patients treated with EMR/ESD according to the lymphatic and venous invasion

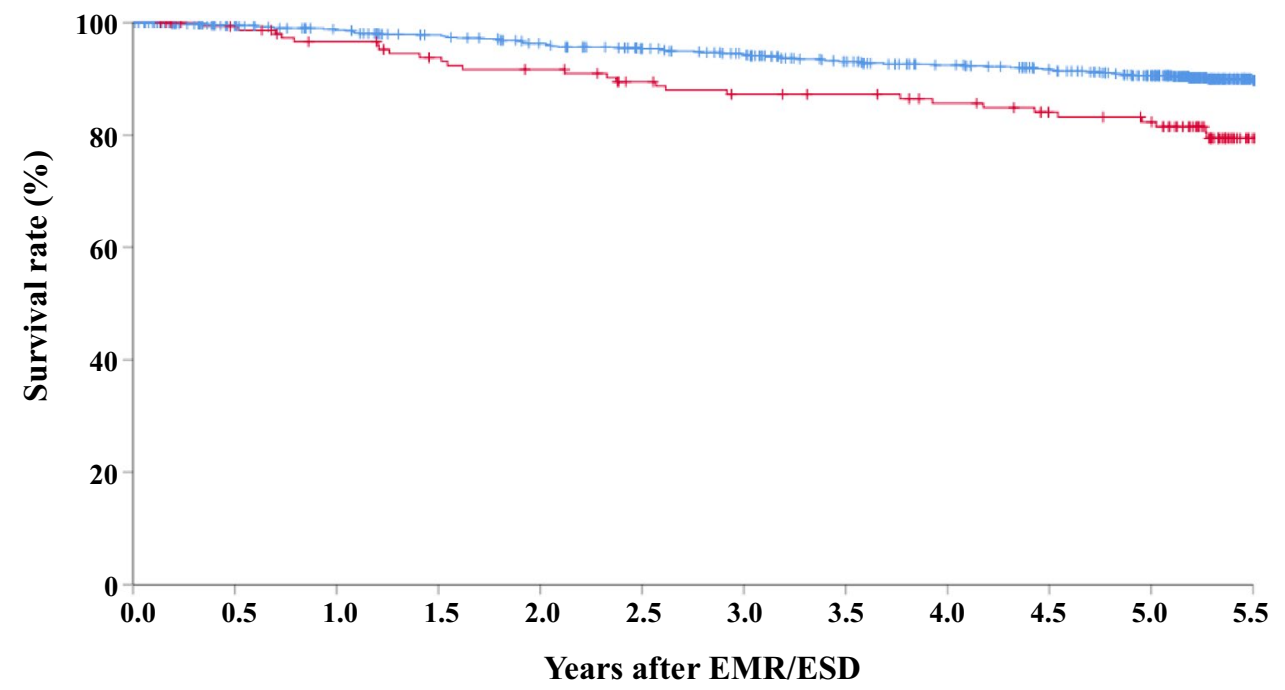

pTla $(\mathrm{n}=817)$ pT1b $(\mathrm{n}=153)$

\begin{tabular}{lccccc}
\hline & \multicolumn{5}{c}{ Years after EMR/ESD } \\
\cline { 2 - 6 } & 1 & 2 & 3 & 4 & 5 \\
\hline pT1a & $98.7 \%$ & $96.3 \%$ & $94.2 \%$ & $92.5 \%$ & $90.6 \%$ \\
pT1b & $96.6 \%$ & $91.7 \%$ & $87.3 \%$ & $85.7 \%$ & $82.4 \%$ \\
\hline
\end{tabular}

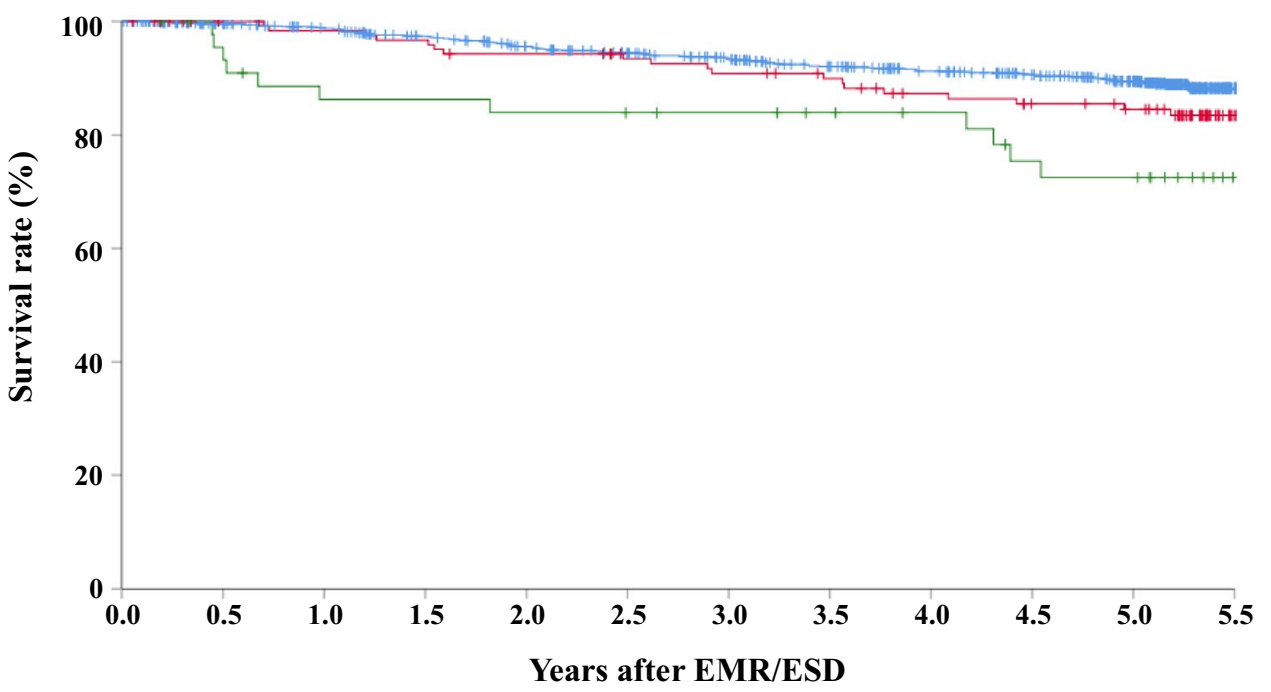

ly- and $v-(n=1032) \quad$ ly + or $v+(n=130) \quad$ Unknown $(n=46)$

\begin{tabular}{lccccc}
\hline & \multicolumn{5}{c}{ Years after EMR/ESD } \\
\cline { 2 - 5 } & 1 & 2 & 3 & 4 & 5 \\
\hline ly- and v- & $98.8 \%$ & $95.6 \%$ & $93.4 \%$ & $91.2 \%$ & $89.5 \%$ \\
ly+ or v+ & $98.4 \%$ & $94.3 \%$ & $90.9 \%$ & $87.4 \%$ & $84.5 \%$ \\
Unknown & $86.5 \%$ & $\mathbf{8 4 . 2} \%$ & $\mathbf{8 4 . 2} \%$ & $\mathbf{8 4 . 2} \%$ & $72.8 \%$ \\
\hline
\end{tabular}




\section{Results in patients treated with chemotherapy}

\section{and/or radiotherapy in 2013}

Tables 12, 13 and Figs. 4, 5, 6.

Table 12 Dose of irradiation (non-surgically treated cases)

\begin{tabular}{lllllll}
\hline Dose of irradiation (Gy) & Definitive & \multicolumn{2}{l}{ Palliative (\%) } & Recurrence (\%) & Others (\%) & Total (\%) \\
\cline { 2 - 5 } & Radiation alone (\%) & With chemotherapy (\%) & & & \\
\hline-29 & $2(1.5 \%)$ & $20(2.4 \%)$ & $23(11.7 \%)$ & $2(5.9 \%)$ & $1(10.0 \%)$ & $48(4.0 \%)$ \\
$30-39$ & $4(3.0 \%)$ & $16(1.9 \%)$ & $33(16.8 \%)$ & $1(2.9 \%)$ & $2(20.0 \%)$ & $56(4.7 \%)$ \\
$40-49$ & $7(5.3 \%)$ & $33(4.0 \%)$ & $40(20.4 \%)$ & $1(2.9 \%)$ & $3(30.0 \%)$ & $84(7.0 \%)$ \\
$50-59$ & $15(11.4 \%)$ & $233(28.2 \%)$ & $35(17.9 \%)$ & $10(29.4 \%)$ & $2(20.0 \%)$ & $295(24.6 \%)$ \\
$60-69$ & $98(74.2 \%)$ & $505(61.1 \%)$ & $63(32.1 \%)$ & $19(55.9 \%)$ & $2(20.0 \%)$ & $687(57.3 \%)$ \\
$70-$ & $6(4.5 \%)$ & $18(2.2 \%)$ & $1(0.5 \%)$ & $1(2.9 \%)$ & $26(2.2 \%)$ \\
Unknown & & $2(0.2 \%)$ & $1(0.5 \%)$ & & $3(0.3 \%)$ \\
Total & 132 & 827 & 196 & 34 & 10 & 1199 \\
Median (min-max) & $60.0(5.4-80.0)$ & $60.0(2.0-99.0)$ & $50.0(2.0-70.0)$ & $60.0(11.0-70.0)$ & $43.2(26.0-66.0)$ & $60.0(2.0-99.0)$ \\
\hline
\end{tabular}

Table 13 Dose of irradiation (surgically treated cases)

\begin{tabular}{lll}
\hline Dose of irradiation (Gy) & $\begin{array}{l}\text { Preoperative } \\
\text { irradiation }(\%)\end{array}$ & $\begin{array}{l}\text { Postoperative irradia- } \\
\text { tion }(\%)\end{array}$ \\
\hline-29 & $4(1.4 \%)$ & $6(10.0 \%)$ \\
$30-39$ & $62(22.2 \%)$ & $3(5.0 \%)$ \\
$40-49$ & $177(63.4 \%)$ & $5(8.3 \%)$ \\
$50-59$ & $19(6.8 \%)$ & $19(31.7 \%)$ \\
$60-69$ & $10(3.6 \%)$ & $24(40.0 \%)$ \\
$70-$ & $4(1.4 \%)$ & $2(3.3 \%)$ \\
Unknown & $3(1.1 \%)$ & $1(1.7 \%)$ \\
Total & 279 & 60 \\
Median (min-max) & $40.0(2.0-99.0)$ & $55.0(16.0-75.9)$ \\
\hline
\end{tabular}


Fig. 4 Survival of patients treated with chemotherapy and/ or radiotherapy

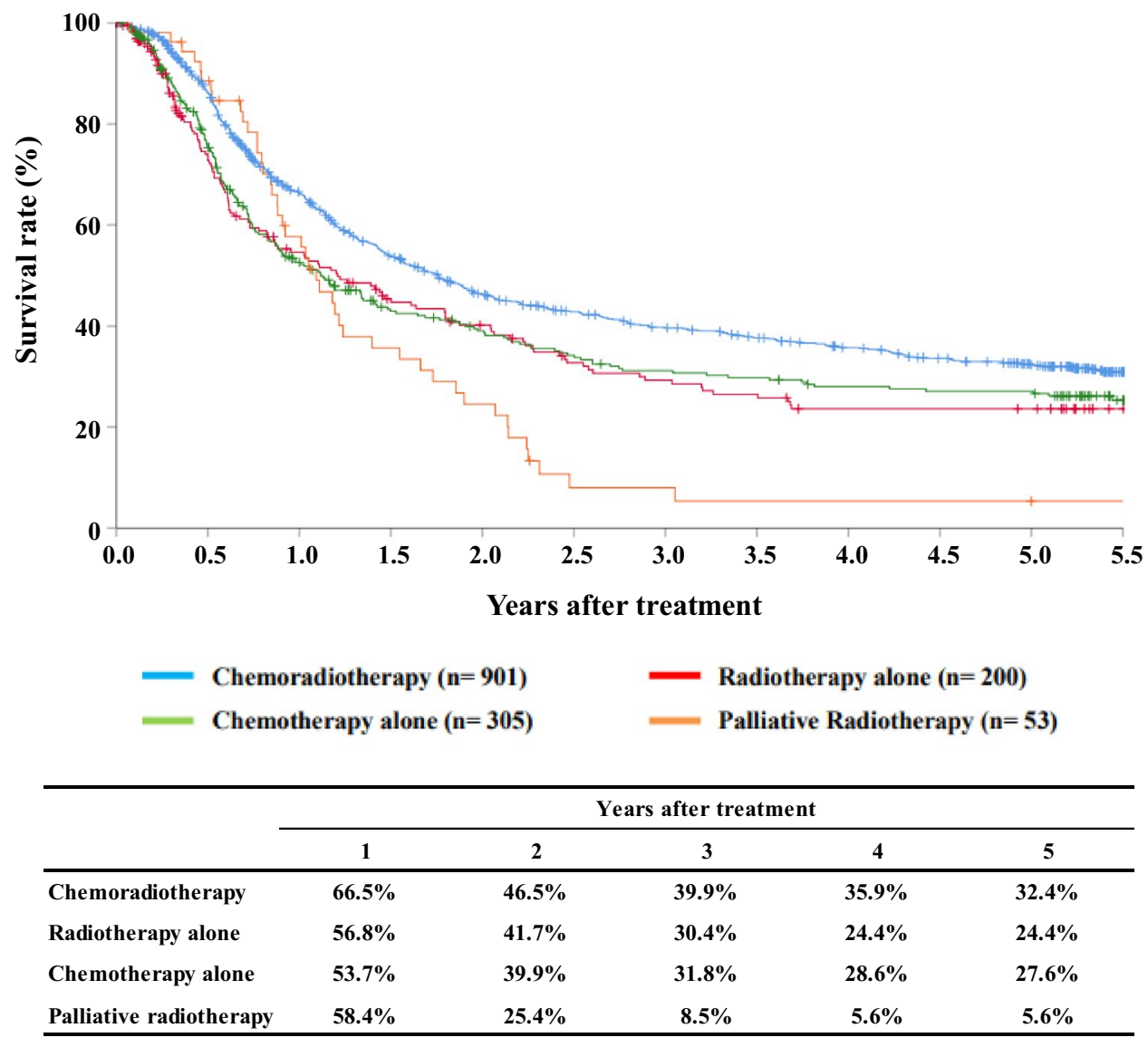


Fig. 5 Survival of patients treated with definitive chemoradiotheraphy according to clinical stage (UICC TNM 7th)

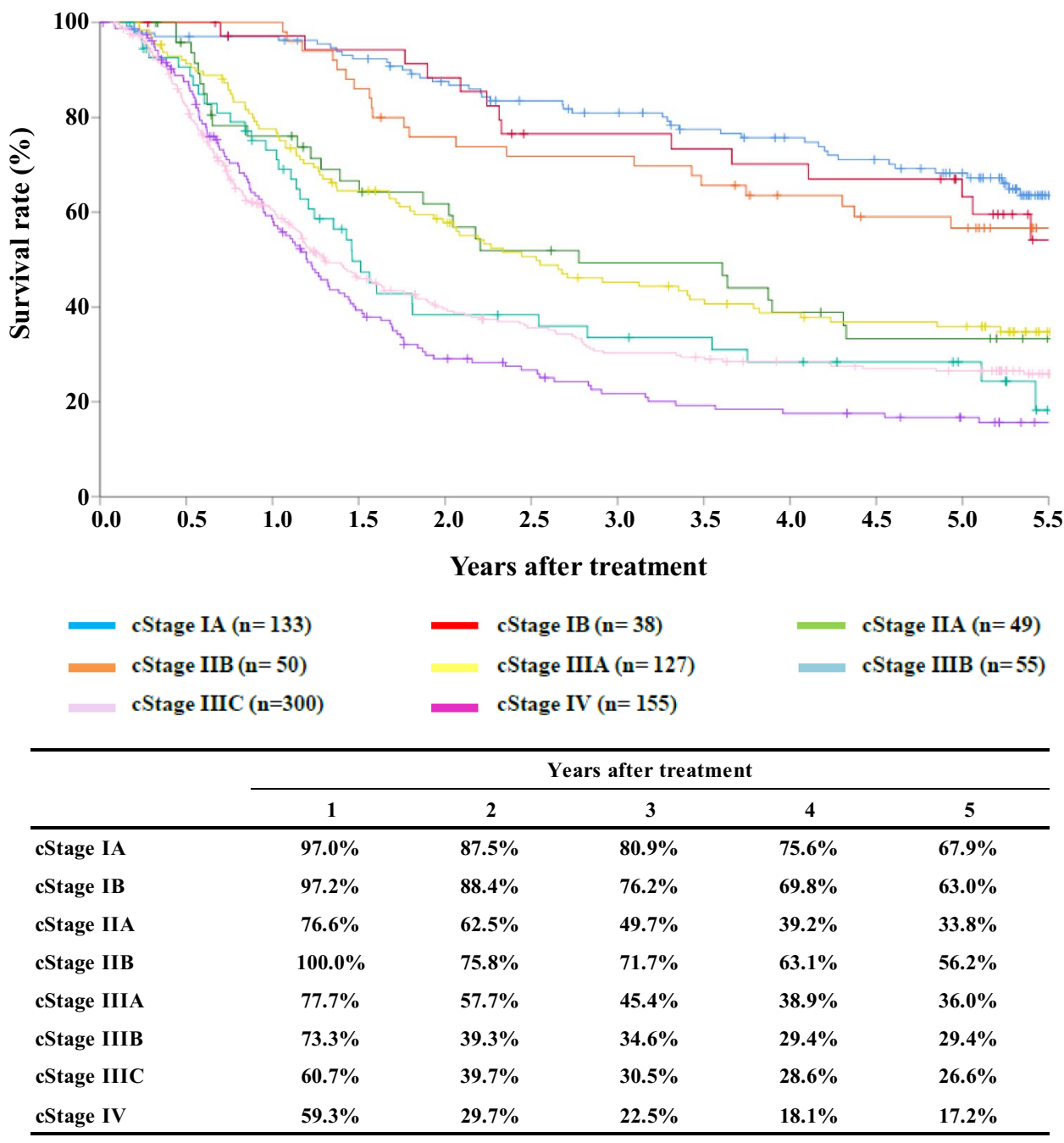


Fig. 6 Survival of patients underwent radiotherapy alone according to clinical stage (UICC TNM 7th)

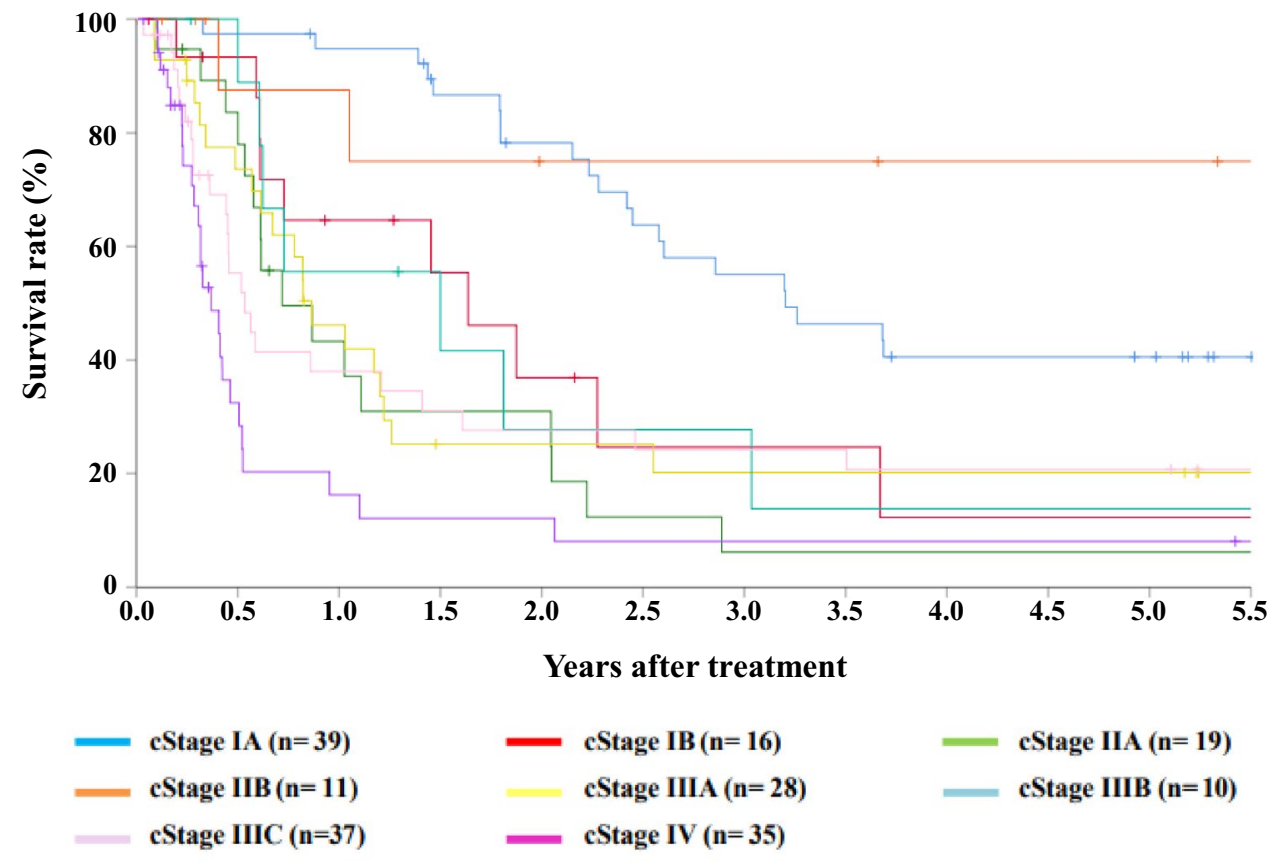

\begin{tabular}{lccccc}
\hline & \multicolumn{3}{c}{ Years after treatment } \\
\cline { 2 - 5 } & 1 & 2 & 3 & 4 & 5 \\
\hline cStage IA & $94.8 \%$ & $78.3 \%$ & $55.1 \%$ & $40.2 \%$ & $40.2 \%$ \\
cStage IB & $65.5 \%$ & $39.3 \%$ & $28.1 \%$ & $14.0 \%$ & $14.0 \%$ \\
cStage IIA & $44.4 \%$ & $31.7 \%$ & $6.3 \%$ & $6.3 \%$ & $6.3 \%$ \\
cStage IIB & $89.5 \%$ & $75.7 \%$ & $75.7 \%$ & $75.7 \%$ & $75.7 \%$ \\
cStage IIIA & $47.2 \%$ & $24.7 \%$ & $19.8 \%$ & $19.8 \%$ & $19.8 \%$ \\
cStage IIIB & $57.9 \%$ & $32.2 \%$ & $32.2 \%$ & $16.1 \%$ & $16.1 \%$ \\
cStage IIIC & $43.3 \%$ & $31.5 \%$ & $27.5 \%$ & $23.6 \%$ & $23.6 \%$ \\
cStage IV & $25.8 \%$ & $19.4 \%$ & $12.9 \%$ & $12.9 \%$ & $12.9 \%$ \\
\hline
\end{tabular}

\section{Results in patients who underwent esophagectomy in 2013}

Tables 14, 15, 16, 17, 18, 19, 20, 21, 22, 23, 24, 25, 26, 27, and Figs. 7, 8, 9, 10, 11, 12, 13, 14, 15

Table 14 Treatment modalities of esophagectomy

\begin{tabular}{ll}
\hline Treatment modalities & Cases $(\%)$ \\
\hline Esophagectomy alone & $2336(47.6 \%)$ \\
Esophagectomy + postoperative chemotherapy & $385(7.8 \%)$ \\
Esophagectomy + postoperative chemoradiotherapy & $109(2.2 \%)$ \\
Esophagectomy + postoperative radiotherapy & $34(0.7 \%)$ \\
Preoperative chemotherapy + esophagectomy & $1558(31.7 \%)$ \\
Preoperative chemoradiotherapy + esophagectomy & $286(5.8 \%)$ \\
Definitive radiotherapy + esophagectomy & $6(0.1 \%)$ \\
Definitive chemoradiotherapy + esophagectomy & $101(2.1 \%)$ \\
Others & $95(1.9 \%)$ \\
Total & 4910 \\
\hline
\end{tabular}

Table 15 Tumor location

\begin{tabular}{ll}
\hline Locations & Cases (\%) \\
\hline Cervical & $166(3.4 \%)$ \\
Upper thoracic & $536(10.9 \%)$ \\
Middle thoracic & $2165(44.1 \%)$ \\
Lower thoracic & $1507(30.7 \%)$ \\
EG & $368(7.5 \%)$ \\
E = G & $85(1.7 \%)$ \\
GE & $72(1.5 \%)$ \\
Unknown & $11(0.2 \%)$ \\
Total & 4910 \\
\hline
\end{tabular}


Table 16 Approaches to tumor resection

\begin{tabular}{ll}
\hline Approaches & Cases (\%) \\
\hline Cervical & $135(2.7 \%)$ \\
Right thoracic & $4171(84.9 \%)$ \\
Left thoracic & $63(1.3 \%)$ \\
Left thoracoabdominal & $115(2.3 \%)$ \\
Abdominal & $171(3.5 \%)$ \\
Transhiatal lower esophagectomy & $94(1.9 \%)$ \\
Transhiatal thoracic esophagectomy & $100(2.0 \%)$ \\
Sternotomy & $2(0.0 \%)$ \\
Others & $46(0.9 \%)$ \\
Unknown & $13(0.3 \%)$ \\
Total & 4910
\end{tabular}

Thoracic includes thoracotomy and thoracoscopic Abdominal includes laparotomy and laparoscopic
Table 17 Video-assisted surgery

\begin{tabular}{ll}
\hline Video-assisted surgery & Cases $(\%)$ \\
\hline None & $2444(49.8 \%)$ \\
Thoracoscopy & $1072(21.8 \%)$ \\
Thoracoscopy + laparoscopy & $1037(21.1 \%)$ \\
Thoracoscopy + laparoscopy + mediastinoscopy & $5(0.1 \%)$ \\
Thoracoscopy + laparoscopy + other & \\
Thoracoscopy + mediastinoscopy & \\
Thoracoscopy + other & $1(0.0 \%)$ \\
Laparoscopy & $237(4.8 \%)$ \\
Laparoscopy + mediastinoscopy & $11(0.2 \%)$ \\
Laparoscopy + mediastinoscopy +other & $11(0.3 \%)$ \\
Mediastinoscopy & $57(1.2 \%)$ \\
Laparoscopy + other & $2(0.0 \%)$ \\
Others & $30(0.6 \%)$ \\
Unknown & $3(0.1 \%)$ \\
Total & 4910
\end{tabular}

Table 18 Fields of lymph node dissection according to the location of tumor

\begin{tabular}{|c|c|c|c|c|c|c|c|c|c|}
\hline $\begin{array}{l}\text { Field of lymphadenec- } \\
\text { tomy }\end{array}$ & Cervical & Upper thoracic & Middle thoracic & Lower thoracic & Abdominal & $\mathrm{E}=\mathrm{G}$ & GE & Unknown & Total \\
\hline None & $5(3.0 \%)$ & $15(2.8 \%)$ & $24(1.1 \%)$ & $31(2.1 \%)$ & $6(1.6 \%)$ & $2(2.4 \%)$ & $2(2.8 \%)$ & $3(27.3 \%)$ & $88(1.8 \%)$ \\
\hline $\mathrm{C}$ & $36(21.7 \%)$ & $8(1.5 \%)$ & $16(0.7 \%)$ & $10(0.7 \%)$ & & & & & $70(1.4 \%)$ \\
\hline $\mathrm{C}+\mathrm{UM}$ & $21(12.7 \%)$ & $1(0.2 \%)$ & $1(0.0 \%)$ & $4(0.3 \%)$ & & & & $1(9.1 \%)$ & $28(0.6 \%)$ \\
\hline $\mathrm{C}+\mathrm{UM}+\mathrm{MLM}$ & $10(6.0 \%)$ & $14(2.6 \%)$ & $47(2.2 \%)$ & $15(1.0 \%)$ & $1(0.3 \%)$ & & & & $87(1.8 \%)$ \\
\hline $\mathrm{C}+\mathrm{UM}+\mathrm{MLM}+\mathrm{A}$ & $69(41.6 \%)$ & $336(62.7 \%)$ & $1098(50.7 \%)$ & $532(35.3 \%)$ & $62(16.8 \%)$ & $10(11.8 \%)$ & $1(1.4 \%)$ & $5(45.5 \%)$ & $2113(43.0 \%)$ \\
\hline $\mathrm{C}+\mathrm{UM}+\mathrm{A}$ & $5(3.0 \%)$ & $4(0.7 \%)$ & $17(0.8 \%)$ & $7(0.5 \%)$ & $1(0.3 \%)$ & & 1 & & $35(0.7 \%)$ \\
\hline $\mathrm{C}+\mathrm{MLM}$ & & & & $1(0.1 \%)$ & & & & & $1(0.0 \%)$ \\
\hline $\mathrm{C}+\mathrm{MLM}+\mathrm{A}$ & $3(1.8 \%)$ & $6(1.1 \%)$ & $14(0.6 \%)$ & $9(0.6 \%)$ & $1(0.3 \%)$ & & & & $33(0.7 \%)$ \\
\hline $\mathrm{C}+\mathrm{A}$ & $3(1.8 \%)$ & $3(0.6 \%)$ & $4(0.2 \%)$ & $6(0.4 \%)$ & & & $1(1.4 \%)$ & & $17(0.3 \%)$ \\
\hline UM & $1(0.6 \%)$ & $2(0.4 \%)$ & $9(0.4 \%)$ & $1(0.1 \%)$ & $1(0.3 \%)$ & & & & $14(0.3 \%)$ \\
\hline $\mathrm{UM}+\mathrm{MLM}$ & $3(1.8 \%)$ & $8(1.5 \%)$ & $41(1.9 \%)$ & $24(1.6 \%)$ & $5(1.4 \%)$ & $1(1.2 \%)$ & & & $82(1.7 \%)$ \\
\hline $\mathrm{UM}+\mathrm{MLM}+\mathrm{A}$ & $3(1.8 \%)$ & $124(23.1 \%)$ & $792(36.6 \%)$ & $668(44.3 \%)$ & $116(31.5 \%)$ & $16(18.8 \%)$ & $10(13.9 \%)$ & & $1729(35.2 \%)$ \\
\hline $\mathrm{UM}+\mathrm{A}$ & & $2(0.4 \%)$ & $12(0.6 \%$ & $9(0.6 \%)$ & $2(0.5 \%)$ & & & & $25(0.5 \%)$ \\
\hline MLM & & $3(0.6 \%)$ & $8(0.4 \%)$ & $8(0.5 \%)$ & $4(1.1 \%)$ & $11.2 \%)$ & $2(2.8 \%)$ & & $26(0.5 \%)$ \\
\hline $\mathrm{MLM}+\mathrm{A}$ & $3(1.8 \%)$ & $4(0.7 \%)$ & $62(2.9 \%)$ & $154(10.2 \%)$ & $141(38.3 \%)$ & $43(50.6 \%)$ & $39(54.2 \%)$ & & $446(9.1 \%)$ \\
\hline A & $4(2.4 \%)$ & $6(1.1 \%)$ & $20(0.9 \%)$ & $28(1.9 \%)$ & $28(7.6 \%)$ & $12(14.1 \%)$ & $16(22.0 \%)$ & $2(18.2 \%)$ & $116(2.4 \%)$ \\
\hline Total & 166 & 536 & 2165 & 1507 & 368 & 85 & 72 & 11 & 4910 \\
\hline
\end{tabular}

$C$ bilateral cervical nodes, $U M$ upper mediastinal nodes, $M L M$ middle-lower mediastinal nodes, $A$ abdominal nodes

Table 19 Reconstruction route

\begin{tabular}{ll}
\hline Route & Cases $(\%)$ \\
\hline None & $62(1.3 \%)$ \\
Subcutaneous & $353(7.2 \%)$ \\
Retrosternal & $1971(40.1 \%)$ \\
Posterior mediastinal & $1972(40.2 \%)$ \\
Intrathoracic & $462(9.4 \%)$ \\
Cervical & $49(1.0 \%)$ \\
Others & $26(0.5 \%)$ \\
Unknown & $15(0.3 \%)$ \\
Total & 4910 \\
\hline
\end{tabular}

Table 20 Organs used for reconstruction

\begin{tabular}{ll}
\hline Organs & Cases $(\%)$ \\
\hline None & $88(1.3 \%)$ \\
Whole stomach & $215(4.3 \%)$ \\
Gastric tube & $4114(83.1 \%)$ \\
Jejunum & $249(5.0 \%)$ \\
Free jejunum & $85(1.7 \%)$ \\
Colon & $162(3.3 \%)$ \\
Free colon & $8(0.2 \%)$ \\
Others & $32(0.6 \%)$ \\
Total organs & 4953 \\
Total cases & 4822 \\
\hline
\end{tabular}


Table 21 Histological classification

\begin{tabular}{ll}
\hline Histological classification & Cases (\%) \\
\hline Squamous cell carcinoma & $4086(83.2 \%)$ \\
Squamous cell carcinoma & $756(15.4 \%)$ \\
Well differentiated & $750(15.3 \%)$ \\
Moderately differentiated & $1989(40.5 \%)$ \\
Poorly differentiated & $591(12.0 \%)$ \\
Adenocarcinoma & $306(6.2 \%)$ \\
Barrett's carcinoma & $118(2.4 \%)$ \\
Adenosquamous carcinoma & $22(0.4 \%)$ \\
Mucoepidermoid carcinoma & $1(0.0 \%)$ \\
Basaloid carcinoma & $86(1.8 \%)$ \\
Neuroendocrine tumor & $1(0.0 \%)$ \\
Neuroendocrine carcinoma & $32(0.7 \%)$ \\
Undifferentiated carcinoma & $8(0.2 \%)$ \\
Malignant melanoma & $16(0.3 \%)$ \\
Carcinosarcoma & $43(0.9 \%)$ \\
GIST & $2(0.0 \%)$ \\
Adenoid cystic carcinoma & $3(0.1 \%)$ \\
Sarcoma & $2(0.0 \%)$ \\
Other carcinomas & $3(0.1 \%)$ \\
Other tumors & $41(0.8 \%)$ \\
Unknown & $140(2.9 \%)$ \\
Total & 4910 \\
\hline &
\end{tabular}

Table 22 Pathological depthe of tumor invasion, pT (JES 10th)

\begin{tabular}{ll}
\hline Pathological depth of tumor invasion & Cases $(\%)$ \\
\hline pTx & $72(1.5 \%)$ \\
pT0 & $161(3.3 \%)$ \\
pT1a & $589(12.0 \%)$ \\
pT1b & $1339(27.3 \%)$ \\
pT2 & $607(12.4 \%)$ \\
pT3 & $1898(38.7 \%)$ \\
pT4a & $138(0.8 \%)$ \\
pT4b & $106(2.2 \%)$ \\
Total & 4910 \\
\hline
\end{tabular}

Table 23 Pathological grading of lymph node metastasis, pN (JES 10th)
Table 24 Pathological grading of lymph node metastasis, $\mathrm{pN}$ (UICC TNM 7th)

Table 25 Pathological findings of distant organ metastasis, $\mathrm{pM}$ (JES 10th)

Table 26 Residual tumor

Table 27 Cause of death

\begin{tabular}{ll}
\hline Cause of death & Cases (\%) \\
\hline Death due to recurrence & $1584(63.8 \%)$ \\
Death due to other cancer & $193(7.8 \%)$ \\
Death due to other disease (with recurrence) & $50(2.0 \%)$ \\
Death due to other disease (without recurrence) & $330(13.3 \%)$ \\
Death due to other disease (recurrence unknown) $^{\text {Operative death }}$ & $15(0.6 \%)$ \\
Postoperative hospital death $^{\mathrm{b}}$ & $38(1.5 \%)$ \\
Unknown $_{\text {Total of death cases }}$ & $59(2.4 \%)$ \\
\hline
\end{tabular}

${ }^{a}$ Operative death means death within 30 days after operation in or out of hospital. Operative mortality rate: $0.77 \%$

${ }^{\mathrm{b}}$ Hospital death is defined as death during the same hospitalization, regardless of department at time of death. Hospital mortality rate: $1.98 \%$

\begin{tabular}{ll}
\hline $\begin{array}{l}\text { Residual tumor } \\
\text { R) }\end{array}$ & Cases $(\%)$ \\
\hline RX & $126(2.6 \%)$ \\
R0 & $4359(88.8 \%)$ \\
R1 & $239(4.9 \%)$ \\
R2 & $186(3.8 \%)$ \\
Total & 4910 \\
\hline
\end{tabular}

\begin{tabular}{ll}
\hline $\begin{array}{l}\text { Lymph node } \\
\text { metastasis }\end{array}$ & Cases (\%) \\
\hline pN0 & $2361(48.1 \%)$ \\
pN1 (1-2) & $1374(28.0 \%)$ \\
pN2 (3-6) & $757(15.4 \%)$ \\
pN3 (7-) & $366(7.5 \%)$ \\
Unknown & $52(1.1 \%)$ \\
Total & 4910 \\
\hline
\end{tabular}

Distant metasta- Cases (\%) sis (M)

\begin{tabular}{ll}
\hline MX & $125(2.5 \%)$ \\
M0 & $4715(96.0 \%)$ \\
M1 & $70(1.4 \%)$ \\
Total & 4910 \\
\hline
\end{tabular}

\begin{tabular}{ll}
\hline $\begin{array}{l}\text { Lymph node } \\
\text { metastasis }\end{array}$ & Cases (\%) \\
\hline pN0 & $2335(47.6 \%)$ \\
pN1 & $936(19.1 \%)$ \\
pN2 & $1000(20.4 \%)$ \\
pN3 & $354(7.2 \%)$ \\
pN4 & $254(5.2 \%)$ \\
Unknown & $31(0.6 \%)$ \\
Total & 4910 \\
\hline
\end{tabular}

\begin{tabular}{|l|r|}
\hline \multicolumn{2}{|c|}{ Follow-up period (months) } \\
\hline Median (min - max) & $59.76(0.33-83.25)$ \\
\hline
\end{tabular}


Fig. 7 Survival of patients who underwent esophagectomy

Fig. 8 Survival of patients who underwent eseophagectomy according to clinical stage (JES 10th)

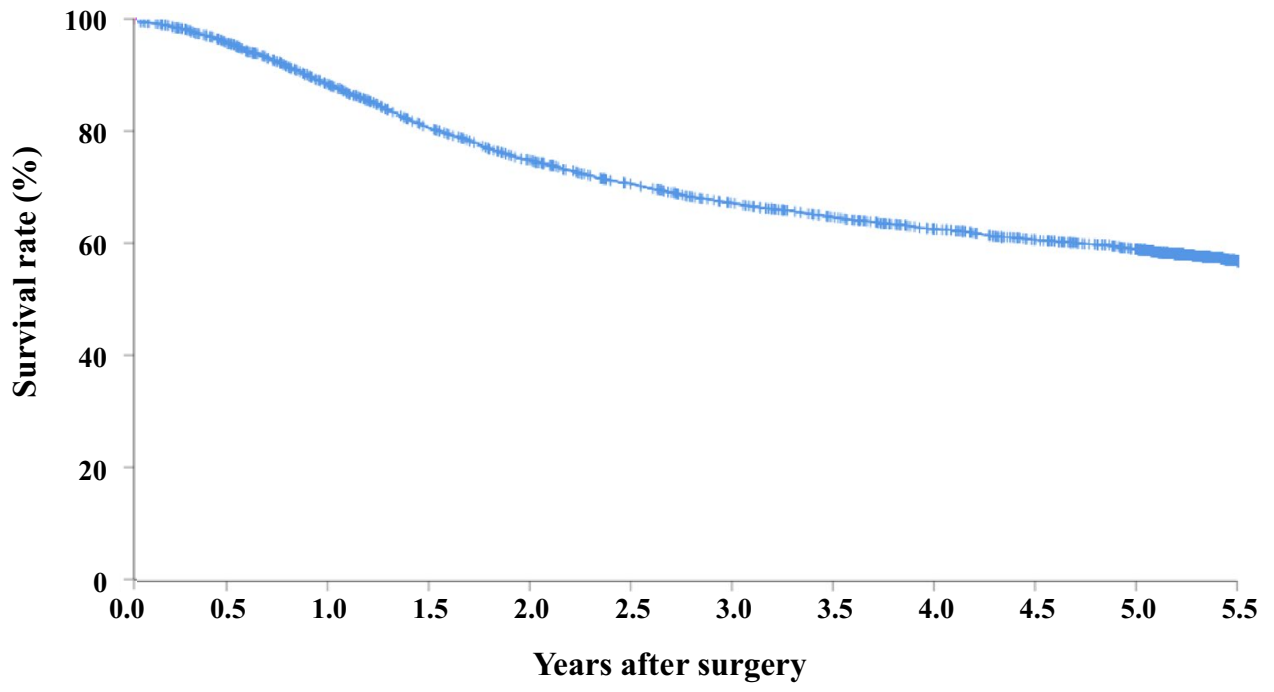

Esophagectomy $(n=4563)$

\begin{tabular}{lcccccc}
\hline & \multicolumn{5}{c}{ Years after surgery } \\
\cline { 2 - 6 } & 1 & 2 & 3 & 4 & 5 \\
\hline Esophagectomy & $88.7 \%$ & $75.2 \%$ & $67.6 \%$ & $62.9 \%$ & $59.3 \%$ \\
\hline
\end{tabular}

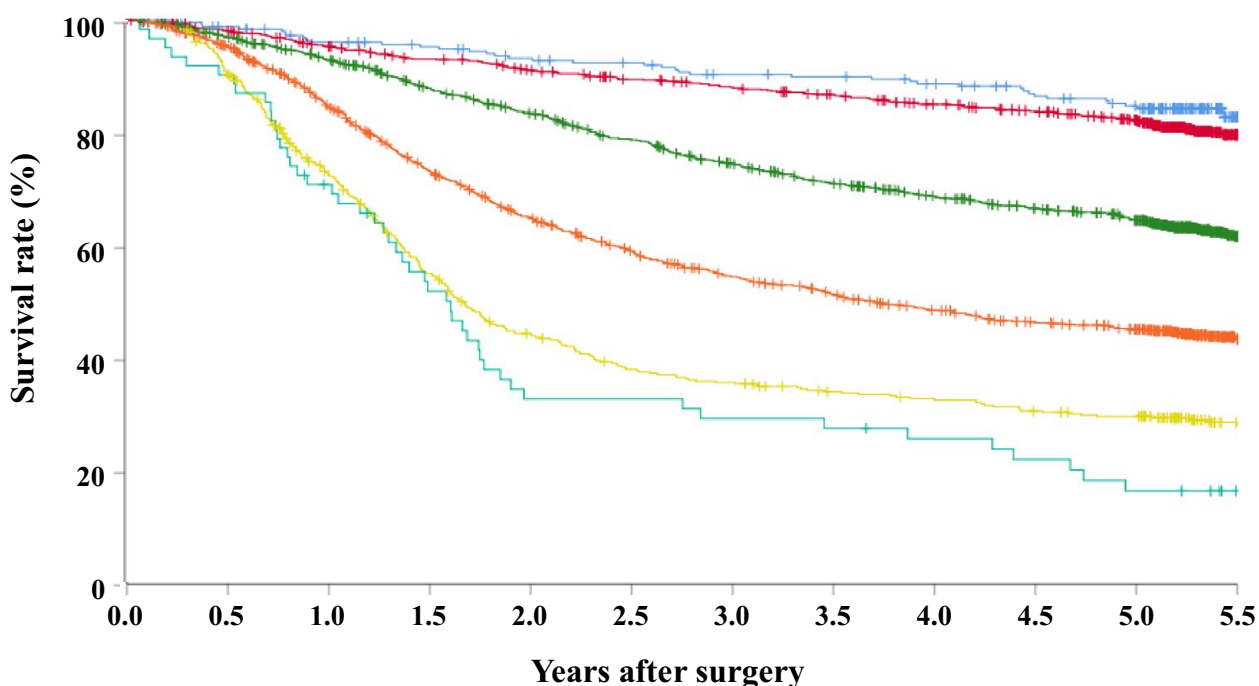

Years after surgery

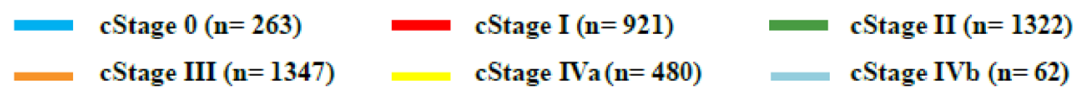

\begin{tabular}{lccccc}
\hline & \multicolumn{5}{c}{ Years after surgery } \\
\cline { 2 - 6 } & 1 & 2 & 3 & 4 & 5 \\
\hline cStage 0 & $96.1 \%$ & $93.3 \%$ & $90.5 \%$ & $88.8 \%$ & $84.9 \%$ \\
cStage I & $95.5 \%$ & $91.1 \%$ & $88.2 \%$ & $85.1 \%$ & $82.4 \%$ \\
cStage II & $93.0 \%$ & $83.6 \%$ & $74.7 \%$ & $68.9 \%$ & $64.6 \%$ \\
cStage III & $84.8 \%$ & $65.3 \%$ & $54.7 \%$ & $48.8 \%$ & $45.3 \%$ \\
cStage IVa & $73.0 \%$ & $44.5 \%$ & $35.9 \%$ & $33.0 \%$ & $29.8 \%$ \\
cStage IVb & $70.5 \%$ & $33.1 \%$ & $29.6 \%$ & $26.0 \%$ & $16.7 \%$ \\
\hline
\end{tabular}


Fig. 9 Survival of patients who underwent esophagectomy according to clinical stage (UICC TNM 7th)

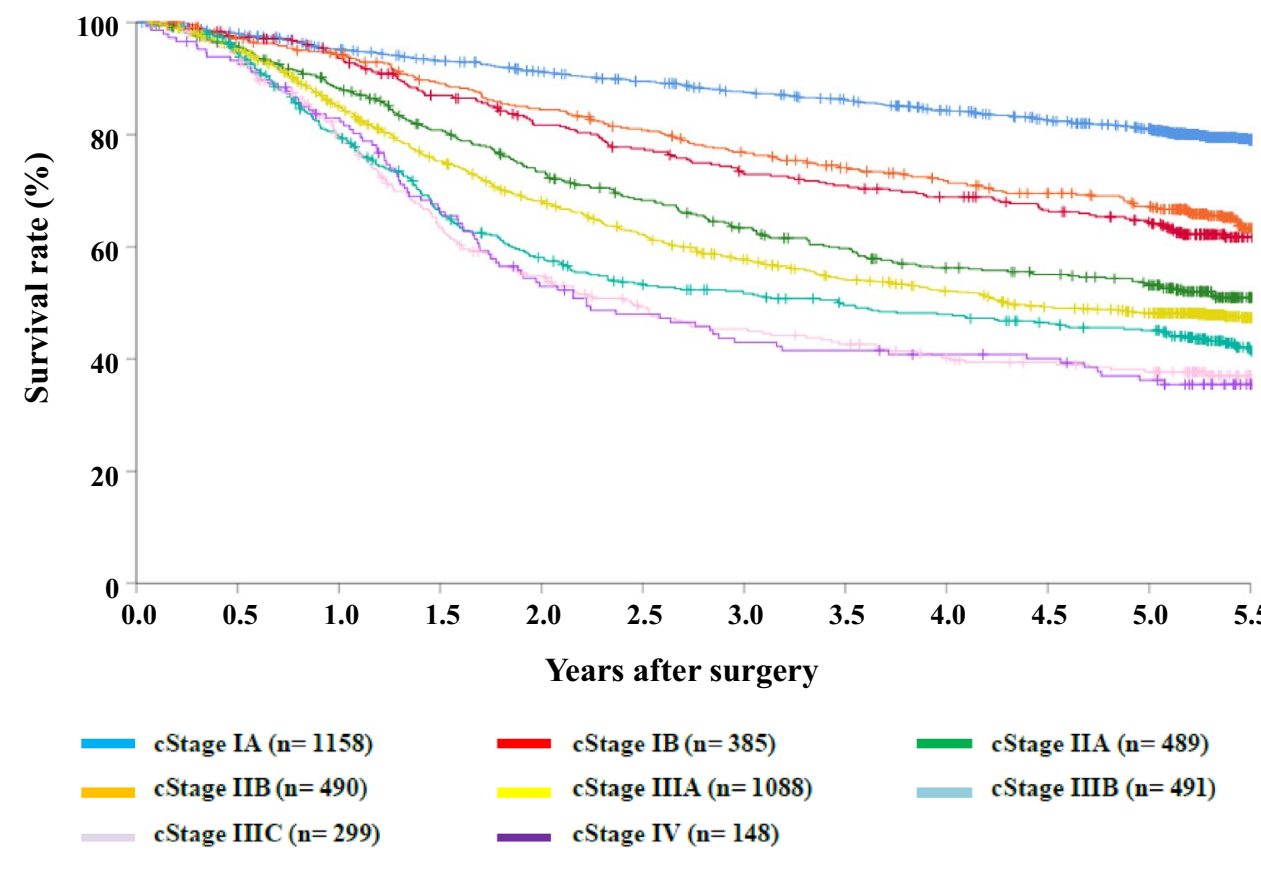

\begin{tabular}{lccccc}
\hline & \multicolumn{3}{c}{ Years after surgery } \\
\cline { 2 - 5 } & 1 & 2 & 3 & 4 & 5 \\
\hline cStage IA & $95.2 \%$ & $91.2 \%$ & $87.6 \%$ & $84.3 \%$ & $81.0 \%$ \\
cStage IB & $93.6 \%$ & $81.8 \%$ & $73.3 \%$ & $69.0 \%$ & $64.5 \%$ \\
cStage IIA & $88.4 \%$ & $73.6 \%$ & $63.5 \%$ & $56.4 \%$ & $53.2 \%$ \\
cStage IIB & $94.2 \%$ & $84.5 \%$ & $77.0 \%$ & $71.9 \%$ & $67.3 \%$ \\
cStage IIIA & $85.0 \%$ & $68.3 \%$ & $57.8 \%$ & $52.2 \%$ & $48.1 \%$ \\
cStage IIIB & $79.9 \%$ & $58.3 \%$ & $51.8 \%$ & $48.1 \%$ & $45.2 \%$ \\
cStage IIIC & $79.1 \%$ & $54.8 \%$ & $45.5 \%$ & $40.3 \%$ & $37.8 \%$ \\
cStage IV & $82.9 \%$ & $53.0 \%$ & $43.0 \%$ & $40.8 \%$ & $36.3 \%$ \\
\hline
\end{tabular}


Fig. 10 Survival of patients who underwent esophagectomy according to the depth of tumor invasion, pT (JES 10th)

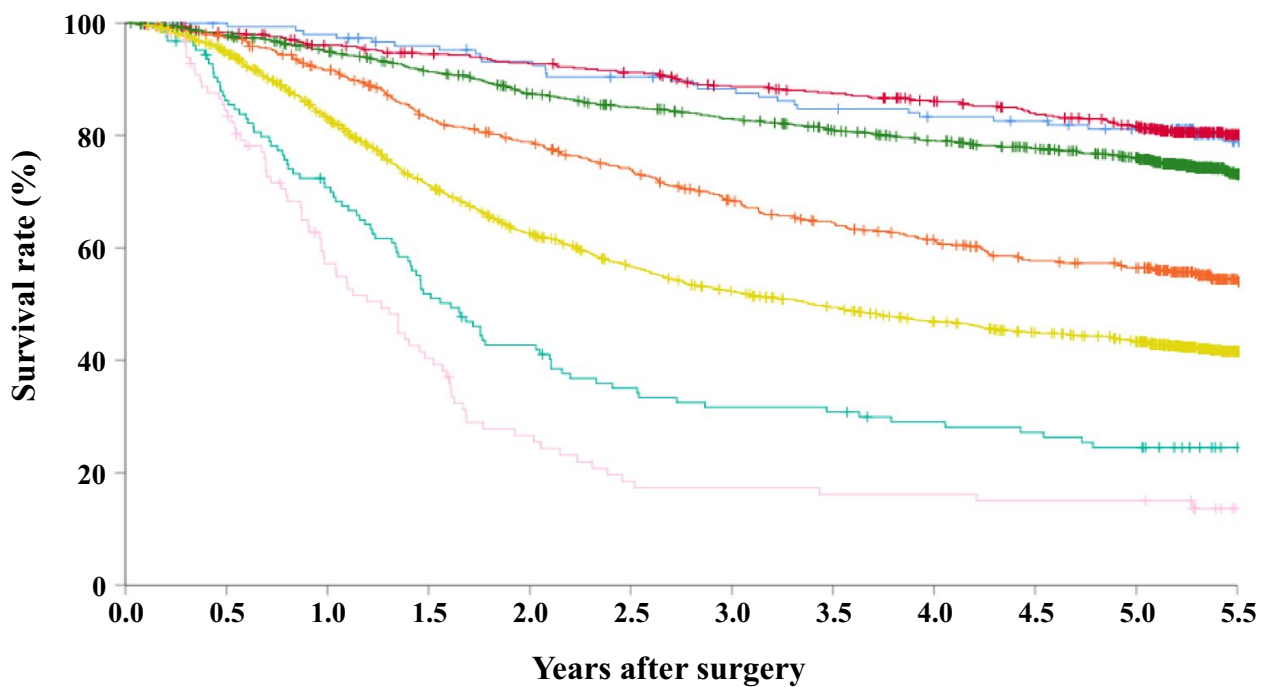

$$
\begin{array}{lll}
\text { pT0 }(n=149) & \text { pTla }(n=540) & \text { pT1b }(n=1246) \\
\text { pT2 }(n=556) & \text { pT3 }(n=1777) & \text { pT4a }(n=125) \\
\text { pT4b }(n=98) & &
\end{array}
$$

\begin{tabular}{lccccc}
\hline & \multicolumn{5}{c}{ Years after surgery } \\
\cline { 2 - 5 } & 1 & 2 & 3 & 4 & 5 \\
\hline pT0 & $98.0 \%$ & $93.2 \%$ & $88.3 \%$ & $83.3 \%$ & $81.1 \%$ \\
pT1a & $96.0 \%$ & $92.8 \%$ & $88.6 \%$ & $86.0 \%$ & $81.6 \%$ \\
pT1b & $95.0 \%$ & $87.4 \%$ & $83.0 \%$ & $79.1 \%$ & $76.0 \%$ \\
pT3 & $91.6 \%$ & $78.9 \%$ & $68.4 \%$ & $61.5 \%$ & $56.4 \%$ \\
pT4a & $83.1 \%$ & $62.8 \%$ & $52.4 \%$ & $47.0 \%$ & $43.4 \%$ \\
pT4b & $70.9 \%$ & $42.7 \%$ & $31.7 \%$ & $29.0 \%$ & $24.5 \%$ \\
\hline
\end{tabular}


Fig. 11 Survival of patients who underwent esophagectomy according to lymph node metastasis (JES 10th)

Fig. 12 Survival of patients who underwent esophagectomy according to lymph node metastasis (UICC TNM 7th)

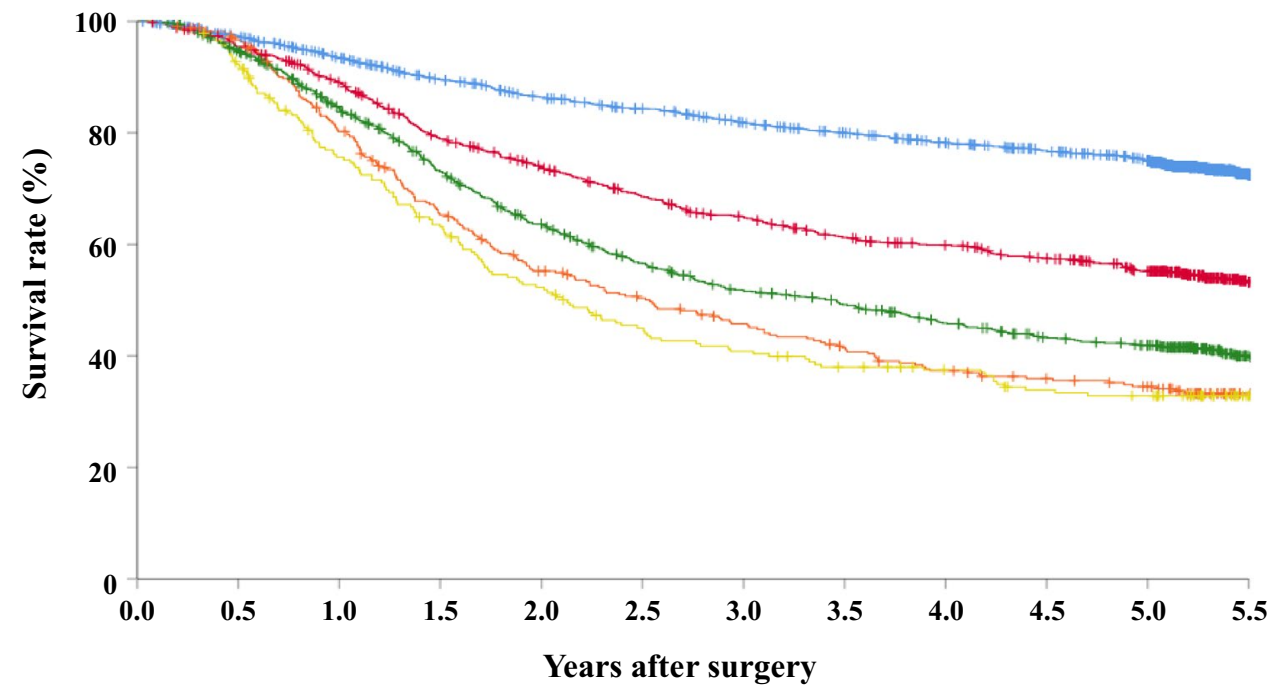

Years after surgery

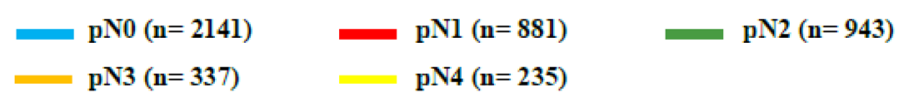

\begin{tabular}{lccccc}
\hline & \multicolumn{5}{c}{ Years after surgery } \\
\cline { 2 - 6 } & 1 & 2 & 3 & 4 & 5 \\
\hline pN0 & $93.5 \%$ & $86.4 \%$ & $81.8 \%$ & $78.2 \%$ & $75.0 \%$ \\
pN1 & $88.9 \%$ & $73.9 \%$ & $64.9 \%$ & $59.8 \%$ & $55.1 \%$ \\
pN2 & $84.3 \%$ & $63.8 \%$ & $51.8 \%$ & $46.0 \%$ & $42.0 \%$ \\
pN4 & $80.4 \%$ & $55.4 \%$ & $46.0 \%$ & $37.5 \%$ & $34.6 \%$ \\
\hline
\end{tabular}

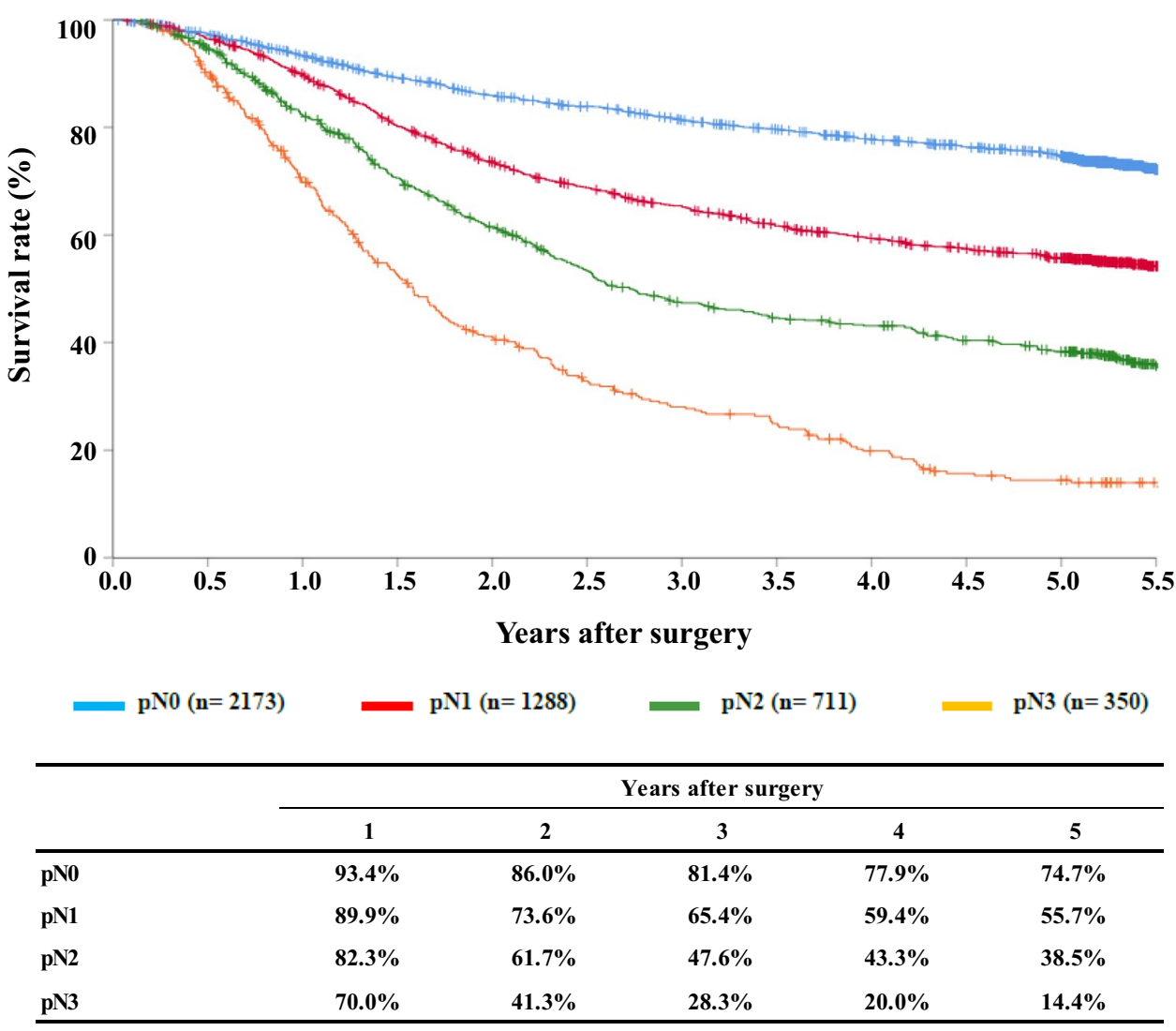


Fig. 13 Survival of patients who underwent esophagectomy according to pathological stage (JES 10th)
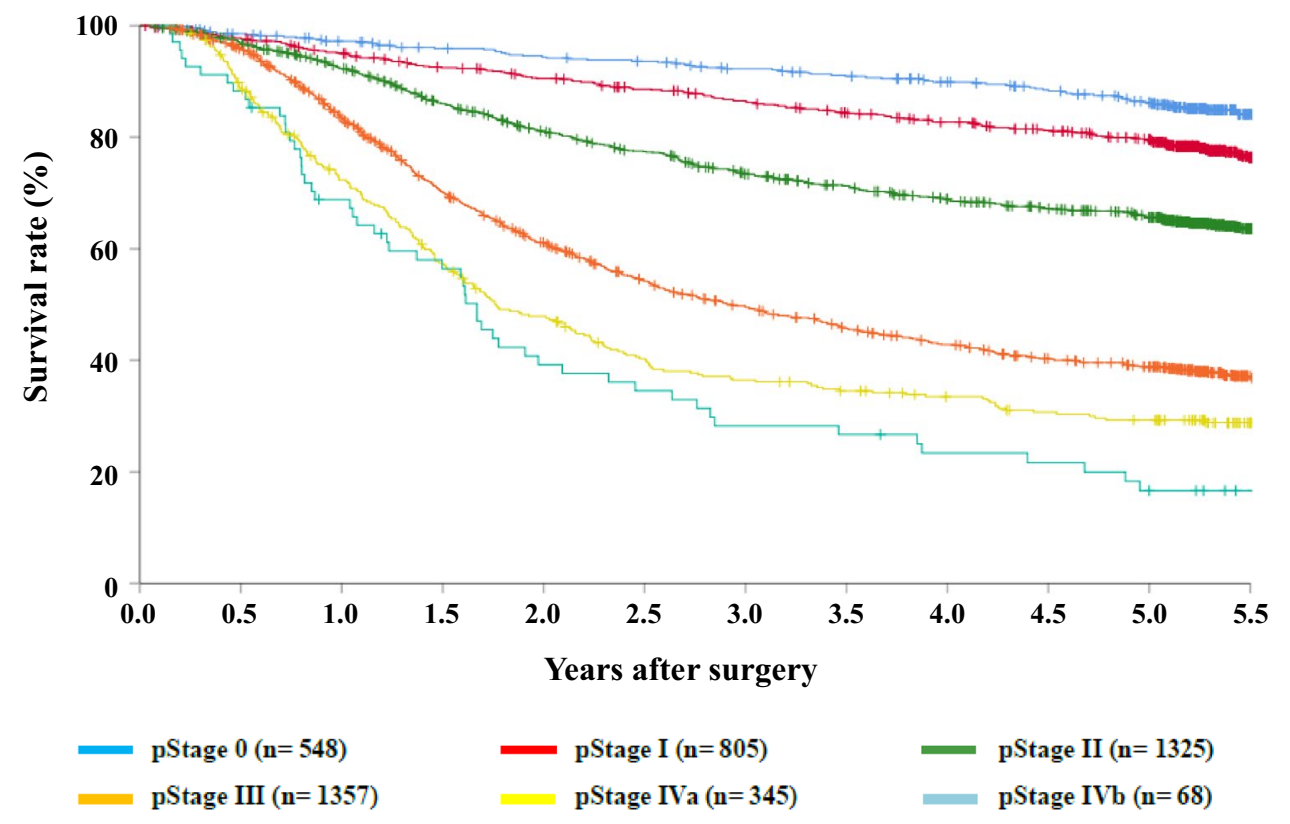

\begin{tabular}{lccccc}
\hline & \multicolumn{5}{c}{ Years after surgery } \\
\cline { 2 - 5 } & 1 & 2 & 3 & 4 & 5 \\
\hline pStage 0 & $97.2 \%$ & $94.4 \%$ & $92.2 \%$ & $89.9 \%$ & $86.2 \%$ \\
pStage I & $95.1 \%$ & $90.5 \%$ & $86.5 \%$ & $82.7 \%$ & $79.5 \%$ \\
pStage II & $92.3 \%$ & $81.0 \%$ & $73.4 \%$ & $68.9 \%$ & $65.6 \%$ \\
pStage III & $83.4 \%$ & $61.3 \%$ & $49.8 \%$ & $42.9 \%$ & $38.9 \%$ \\
pStage IVa & $72.5 \%$ & $47.9 \%$ & $36.6 \%$ & $33.6 \%$ & $29.3 \%$ \\
pStage IVb & $68.7 \%$ & $39.3 \%$ & $28.3 \%$ & $23.5 \%$ & $16.5 \%$ \\
\hline
\end{tabular}


Fig. 14 Survival of patients who underwent esophagectomy according to pathological stage (UICC TNM 7th)

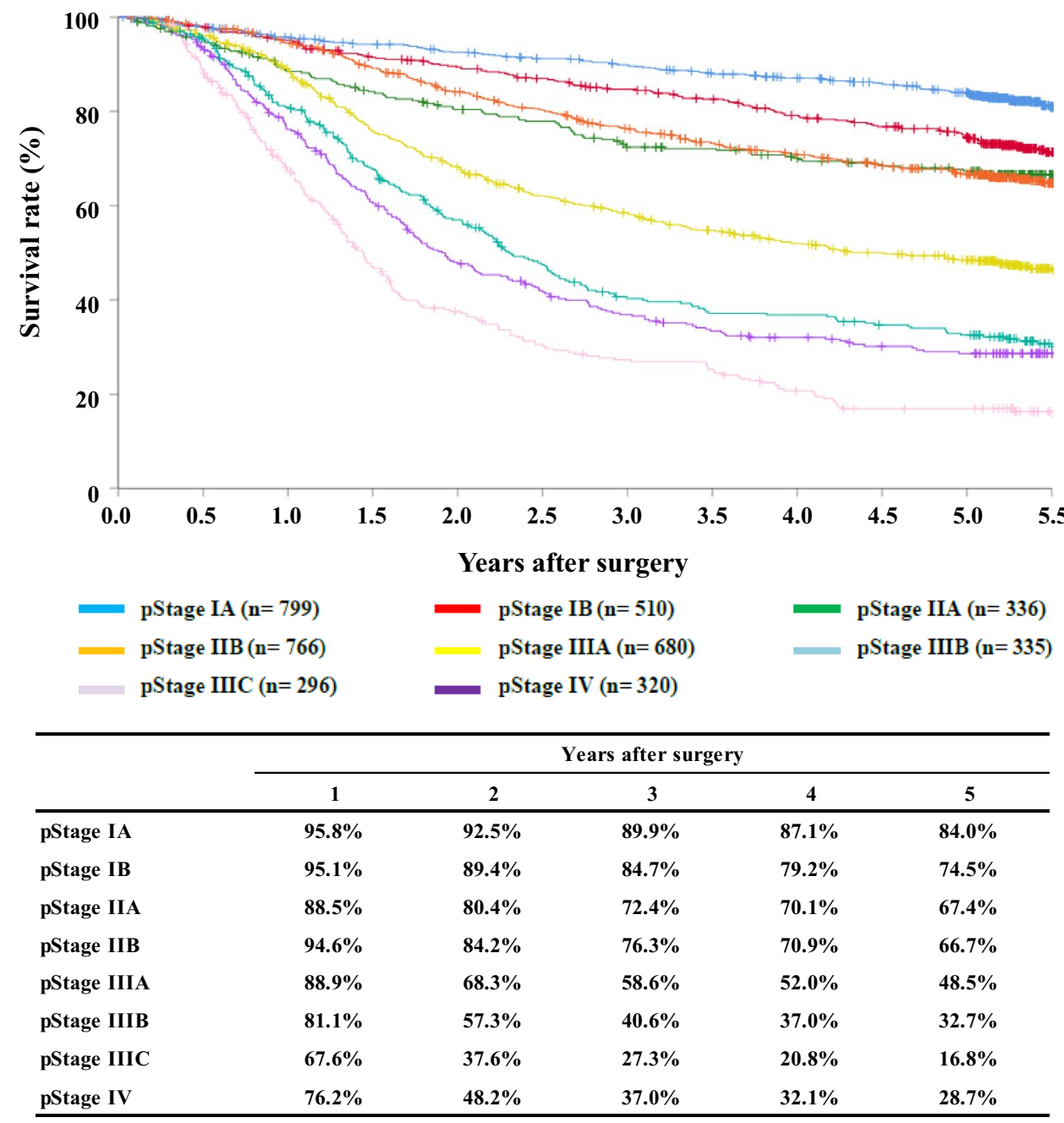


Fig. 15 Survival of patients who underwent esophagectomy according to residual tumor $(\mathrm{R})$

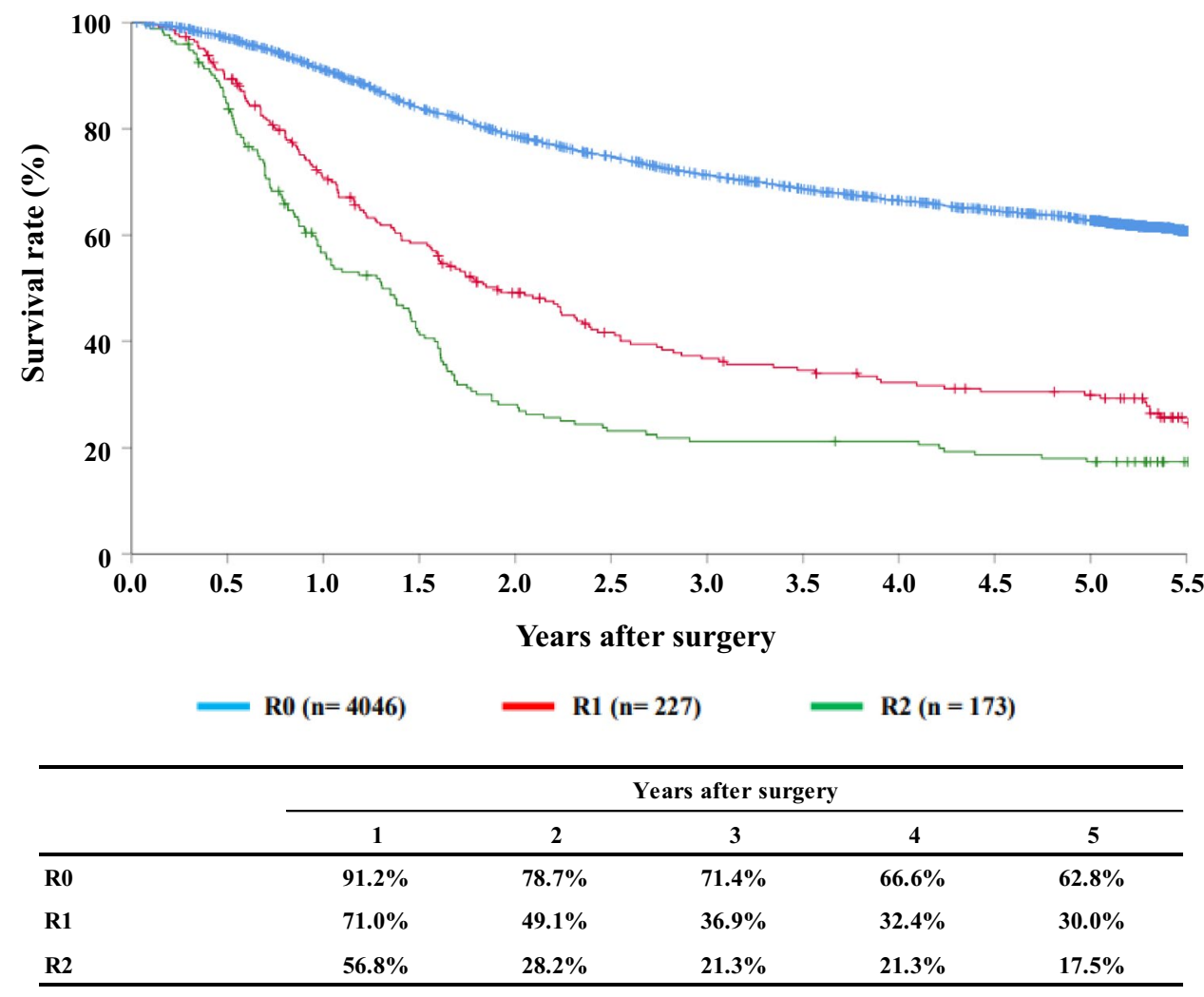

\section{Compliance with ethical standards}

Conflict of interest All authors have nothing to disclose with regard to commercial support.

Ethical statement All procedures followed in accordance with the ethical standards of the responsible committee on human experimentation (institutional and national) and with the Helsinki Declaration of 1964 and later versions.

Open Access This article is licensed under a Creative Commons Attribution 4.0 International License, which permits use, sharing, adaptation, distribution and reproduction in any medium or format, as long as you give appropriate credit to the original author(s) and the source, provide a link to the Creative Commons licence, and indicate if changes were made. The images or other third party material in this article are included in the article's Creative Commons licence, unless indicated otherwise in a credit line to the material. If material is not included in the article's Creative Commons licence and your intended use is not permitted by statutory regulation or exceeds the permitted use, you will need to obtain permission directly from the copyright holder. To view a copy of this licence, visit http://creativecommons.org/licenses/by/4.0/.

\section{Reference}

1. Japan Esophageal Society. Japanese classification of esophageal cancer, 10th edition: part 1. Esophagus. 2009;6:1-25.

2. Sobin LH, Gospodarowicz MK, Wittekind C, UICC International Union Against Cancer. TNM classification of malignant tumors. 7th ed. New York: Wiley-Blackwell; 2009.

Publisher's Note Springer Nature remains neutral with regard to jurisdictional claims in published maps and institutional affiliations. 


\section{Affiliations}

\section{Masayuki Watanabe ${ }^{1}$ (D) $\cdot$ Yuji Tachimori ${ }^{2} \cdot$ Tsuneo Oyama $^{3} \cdot$ Yasushi Toh $^{4} \cdot$ Hisahiro Matsubara $^{5} \cdot$ Masaki Ueno $^{6}$. Koji Kono ${ }^{7}$ Takashi Uno ${ }^{8} \cdot$ Ryu Ishihara ${ }^{9} \cdot K_{\text {Kei Muro }}{ }^{10} \cdot$ Hodaka Numasaki $^{11} \cdot$ Koji Tanaka $^{12} \cdot$ Soji Ozawa $^{13}$.

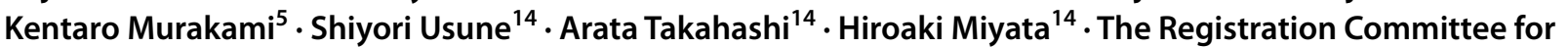 Esophageal Cancer of the Japan Esophageal Society}

1 Department of Gastroenterological Surgery, Cancer Institute Hospital of Japanese Foundation for Cancer Research, Tokyo, Japan

2 Cancer Care Center, Kawasaki Saiwai Hospital, Kawasaki, Kanagawa, Japan

3 Department of Endoscopy, Saku Central Hospital Advanced Care Center, Saku, Nagano, Japan

4 Department of Gastroenterological Surgery, National Hospital Organization Kyushu Cancer Center, Fukuoka, Japan

5 Department of Frontier Surgery, Graduate School of Medicine, Chiba University, Chiba, Japan

6 Department of Gastroenterological Surgery, Toranomon Hospital, Tokyo, Japan

7 Department of Gastrointestinal Tract Surgery, Fukushima Medical University School of Medicine, Fukushima, Japan
8 Department of Diagnostic Radiology and Radiation Oncology, Graduate School of Medicine, Chiba University, Chiba, Japan

9 Department of Gastrointestinal Oncology, Osaka International Cancer Institute, Osaka, Japan

10 Department of Clinical Oncology, Aichi Cancer Center Hospital, Nagoya, Japan

11 Department of Medical Physics and Engineering, Graduate School of Medicine, Osaka University, Suita, Osaka, Japan

12 Department Gastroenterological Surgery, Graduate School of Medicine, Osaka University, Osaka, Japan

13 Department of Gastroenterological Surgery, Tokai University School of Medicine, Isehara, Kanagawa, Japan

14 Department of Healthcare Quality Assessment, Graduate School of Medicine, The University of Tokyo, Tokyo, Japan 